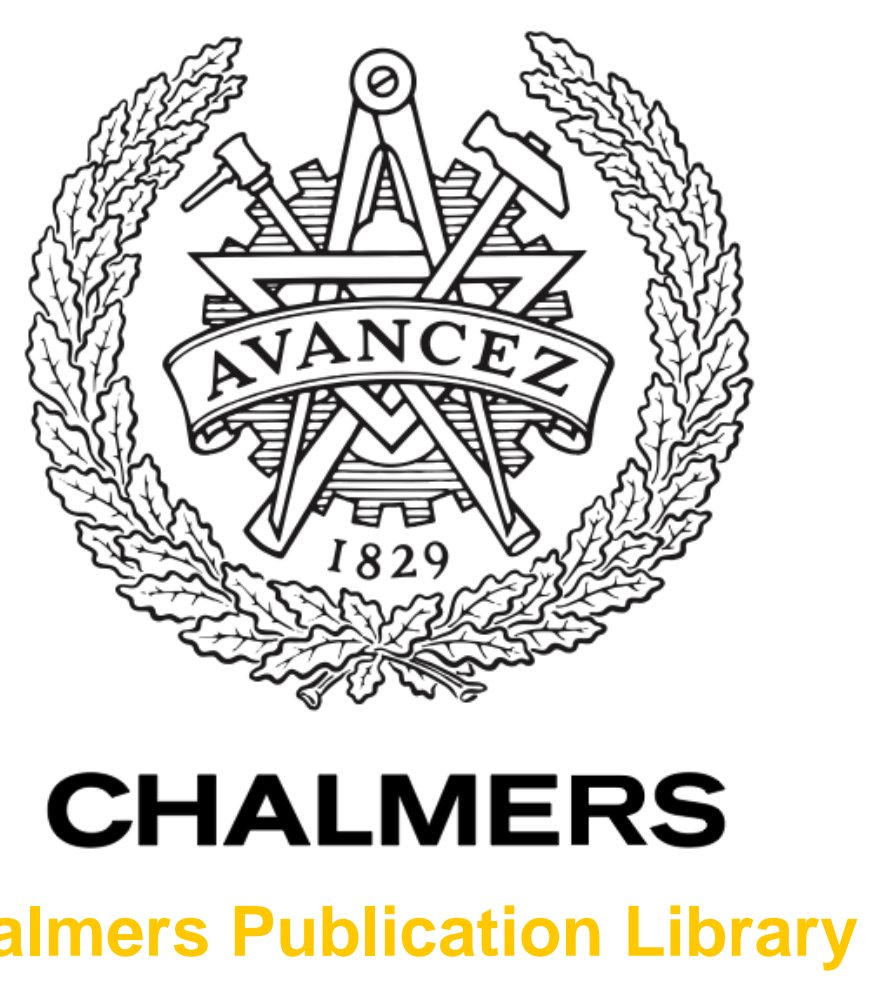

Chalmers Publication Library

\title{
Numerical simulations of Boger fluids through different contraction configurations for the development of a measuring system for extensional viscosity
}

This document has been downloaded from Chalmers Publication Library (CPL). It is the author's version of a work that was accepted for publication in:

Rheologica Acta (ISSN: 0035-4511)

Citation for the published paper:

Nyström, M. ; Jahromi, H. ; Stading, M. (2012) "Numerical simulations of Boger fluids through different contraction configurations for the development of a measuring system for extensional viscosity". Rheologica Acta, vol. 51(8), pp. 713-727.

http://dx.doi.org/10.1007/s00397-012-0631-0

Downloaded from: http://publications.lib.chalmers.se/publication/163649

Notice: Changes introduced as a result of publishing processes such as copy-editing and formatting may not be reflected in this document. For a definitive version of this work, please refer to the published source. Please note that access to the published version might require a subscription. 


\title{
Numerical simulations of Boger fluids through different contraction configurations for the development of a measuring system for extensional viscosity
}

\author{
M. NySTRÖM ${ }^{1}$, H.R. TAMADDON JAHROMI ${ }^{2}$, M. StADING1 ${ }^{, 3}$ AND M.F. WEBSTER ${ }^{2}$ \\ ${ }^{1}$ SIK - The Swedish Institute for Food and Biotechnology, Structure and Material \\ Design, SE-40229 Göteborg, Sweden \\ ${ }^{2}$ Institute of Non-Newtonian Fluid Mechanics, Swansea University, College of \\ Engineering, Swansea, SA28PP, UK \\ ${ }^{3}$ Department of Materials and Manufacturing Technology, Chalmers University of \\ Technology, SE-412 76 Göteborg, Sweden
}

\begin{abstract}
This paper reports the flow behaviour of Newtonian and Boger fluids through various axisymmetric contraction configurations by means of numerical predictions. A principal aim has been to evaluate the geometrical design choice of the hyperbolic contraction flow. The FENE-CR model has been used to reflect the behaviour of Boger fluids, with constant shear viscosity, finite (yet large) extensional viscosity and less than quadratic first normal stress difference. Numerical calculations have been performed on six different contraction configurations to evaluate an optimized geometry for measuring extensional viscosity in uniaxial extensional flow. The influence of a sharp or rounded recess-corner on the nozzle has also been investigated. Few commercial measuring systems are currently available for measurement of the extensional rheology of medium-viscosity fluids, such as foods and other biological systems. In this context, a technique based on the hyperbolic contraction flow would be a suitable alternative. The pressure drop, the velocity field, the first normal stress difference and the strain rate across the geometry have each been evaluated for Newtonian and Boger fluids. This numerical study has shown that the hyperbolic configuration is superior to the other geometry choices in achieving a constant extension rate. In this hyperbolic configuration, no vortices are formed, the measuring range is broader and the strain rate is constant throughout the geometric domain, unlike in the alternative configurations tested. The difference between sharp and rounded recess-corner configurations proved to be negligible and a rise in excess pressure drop (epd) for increasing deformation rates has been observed.
\end{abstract}

Keywords: Viscoelastic fluid; Extensional viscous flow; hyperbolic contraction flow; pressure-drop 


\section{Introduction}

Shear flow has traditionally been considered to be the significant deformation around which rheometric instrumentation must be constructed. However, any change in geometry during the processing of a non-Newtonian, viscoelastic liquid generates a flow with an extensional component. The extensional response, described by the extensional viscosity can differ considerably from the shear response, making it an important factor in processing, product development and quality control of products. The extensional properties during processing have been given increasingly more attention and further important applications are being discovered. Experimental determination of extensional viscosity is still a challenging task even though the research on extensional flow has progressed. Although various experimental techniques have been developed to measure extension, e.g. filament stretching (Sridhar et al. 1991), the Meissner elongational melt rheometer (Meissner 1972; Meissner and Hostettler 1994), capillary break-up (Entov and Hinch 1997) and opposed jets (Fuller et al. 1985), only the capillary break-up method (CABER) is currently available on the commercial market. While these techniques are suitable for either polymer melts or dilute fluids, an efficient technique for medium viscosity fluids (fluids in between dilute solutions and more solid-like systems such as polymer melts) is still lacking.

Food systems are often viscoelastic with medium viscosities, and development of a technique that is able to determine extensional properties of food systems for quality control, product development and design is highly desirable. It would also be attractive to develop measurement systems that are capable of measuring at high strains and strain rates. Several simulations and experimental investigations have been performed to study extension in contraction flows, which would prove to be a suitable measuring system for determining the extensional properties of medium range viscosity fluids, like as some foodstuffs. The importance of being able to measure extensional properties in food science is its significant impact on sensory perception, texture and mouth feel and a deeper understanding of the extensional properties makes it possible to structurally engineer a desired texture and the behaviour of new food products. 
Contraction flow through a hyperbolic nozzle has been suggested as a suitable system for measuring extensional properties for fluids such as foods (e.g. dough), dispersions and medical systems (Oom et al. 2008) by Wikström and Bohlin (Wikström and Bohlin 1999a) and Stading and Bohlin (Stading and Bohlin 2001, 2000). Other groups that have utilized a hyperbolic nozzle in measuring extensional flow are Baird et al (Baird and Huang 2006; Baird 2010), Collier at al. (Collier et al. 1998; Kim et al. 1994) and James et al. (James et al. 1990), although these studies were for polymer melts.

The complex flow arising in contraction flows containing both shear and extensional flow has received considerable attention over many years, see for instance (Boger 1987), (Binding et al. 1998) and review papers (Walters and Webster 2003) and (White et al. 1987). Regions with strong shearing are found along the wall and there is a region with non-homogenous uniaxial extensional flow at the centre of the flow. The aim of this work has been to numerically study the shear and extensional flow in different geometrical configurations to find an optimal geometry for achieving a reasonably constant uniaxial extensional flow, where there is small impact of shear flow on the measuring system, suitable for fluids in the medium viscosity range. Newtonian and Boger fluids has been used as model fluids to extract the extensional and shear behaviour in the contractions.

A further aim has been to validate the assumptions made in the work by Wikström, Bohlin and Stading (Stading and Bohlin 2001, 2000; Wikström and Bohlin 1999a), based on Bindings work (Binding 1988). There, the extensional properties were determined based on calculations compensating for shear flow by assuming a simple Power law model. Here, the flow of constant viscosity viscoelastic fluids through several different axisymmetric contraction configurations has been analysed through strain and shear rate profiles, pressure drops and $\mathrm{N}_{1}$ profiles. This study reveals how maximum extensional viscosity can be extracted from specific geometry choices (hyperbolic) by avoiding the creation of vortices. In this manner, the stream traces of the different geometrical configurations are also investigated. 


\section{Governing equations and flow problem}

In this study, numerical simulations of the flow of Newtonian and viscoelastic Boger fluids through different contractions were considered over a wide range of Weissenberg numbers (We-numbers). The FENE-CR model (Chilcott and Rallison 1988), which displays a constant shear viscosity and an almost quadratic first normal stress difference $\left(N_{l}\right)$ was employed as a representation of a Boger fluid. The material functions used in the study are summarized in table 1. The numerical algorithm was a hybrid finite element/finite volume method(fe/fv(sc)), which is based on a Taylor-Galerkin (Hawken et al. 1990) and a pressurecorrection methodology, as presented by Wapperom and Webster (Wapperom and Webster 1999).

\section{Governing field equations}

The relevant equations used to describe the flow of an isothermal, incompressible viscoelastic fluid are the equations of continuity and motion expressed as:

$$
\begin{gathered}
\nabla \cdot u=0 \\
\operatorname{Re}\left(\frac{\partial u}{\partial t}+u \times \nabla u\right)=-\nabla p+\nabla \times(\tau+2 \beta D)
\end{gathered}
$$

Here, $u$ is the velocity vector, $t$ the time, $p$ the hydrodynamic pressure, $D$ the rateof-deformation $\left(\nabla u+\nabla u^{T}\right) / 2$ and $\tau$ the total extra stress tensor. $\beta$ is the solventviscosity ratio parameter $\beta=\eta_{s} /\left(\eta_{s}+\eta_{p}\right)$, where $\eta_{s}$ is the solvent viscosity and $\eta_{p}$ is the polymeric viscosity. Then, $\eta_{0}$ is defined as the zero-shear-viscosity $\eta_{0}=\eta_{p}+\eta_{s}$ (characteristic viscosity scale). The non-dimensional Re-number is defined as: $\operatorname{Re}=\frac{\rho U_{\text {avg }} L}{\eta_{s}+\eta_{p}}$, where $\rho, L$ and $U_{\text {avg }}$ are the density, the contraction gap width and the characteristic velocity (average upstream velocity), respectively. In 
this study, creeping flow is assumed $\left(\operatorname{Re} \approx 0\left(10^{-2}\right)\right)$ and as a result, the momentum convection term contribution is negligible.

A further non-dimensional parameter of interest for the flow is the Weissenberg number, which is defined as $W e=\lambda U / L$, and depends on the characteristic relaxation time of the fluid $(\lambda)$, density $(\rho)$, characteristic velocity scale $\left(U=U_{\text {avg }}\right)$ and length scale $(L)$. Throughout, the results of this study are presented nondimensionally, with in addition to the above scales that on time also of $(U / L)$.

\section{Constitutive equation and flow problem}

The FENE-CR (Finitely Extensible Nonlinear Elastic-Chilcott Rallison) model (Chilcott and Rallison 1988) has been chosen as an appropriate constitutive model for modelling Boger fluids and Newtonian solutions were taken as a based reference for the viscoelastic calculations. The reason for selecting the FENE-CR model is that it predicts a constant shear viscosity. The first normal stress difference $\left(N_{l}\right)$ of the FENE-CR model is weaker than the strong quadratic form exhibited by the Oldroyd-B model, see Fig. 1a. Furthermore, unlike the OldroydB model the FENE-CR model does not encounter a singularity in extensional viscosity (hence, exhibiting finite extensional response). The constitutive equation for the FENE-CR is expressed as:

$$
f(\operatorname{Tr}(A)) A+W e \stackrel{\nabla}{A}-f(\operatorname{Tr}(A)) I=0,
$$

where $A$ is a stress conformation tensor and the stress is calculated by the conformation transformation:

$$
\tau=\frac{(1-\beta) f(\operatorname{Tr}(\boldsymbol{A}))(\boldsymbol{A}-I)}{W e} .
$$

$f(\operatorname{Tr}(A))$ is the stretch function, which is dependent on the Hookean-dumbbell chain-length extensibility parameter $L$, the trace operator $\operatorname{Tr}(A)$ and the identity tensor $I$ as: 


$$
f(\operatorname{Tr}(\boldsymbol{A}))=\frac{1}{1-\operatorname{Tr}(\boldsymbol{A}) / L^{2}} .
$$

The associated rheometrical functions are given by

$$
\begin{aligned}
& \eta=\eta_{0}, \\
& \mathrm{~N}_{1}=\frac{2 \eta_{0}(1-\beta) W e \dot{\gamma}^{2}}{f^{2}}, \quad \mathrm{~N}_{2}=0, \\
& \eta_{e}=3 \beta \eta_{0}+3(1-\beta) \eta_{0}\left[\frac{f}{f^{2}-f W e \dot{\varepsilon}-2(W e \dot{\varepsilon})^{2}}\right],
\end{aligned}
$$

where $f=f(\operatorname{Tr}(A))$ is defined in Eq. 5 .

The limiting level of the extensional viscosity plateau in the model can be adjusted by changing the extensibility parameter, $L$, (so-called Hookean-dumbbell chain-length extensibility parameter) which measures the size of the polymer molecule in relation to its equilibrium size. Then, an increasing $L$ parameter yields an increased plateau level approaching the Oldroyd-B model for high $L$-levels, Fig. 1b. In this study, the L-parameter has been set to the relatively modest level of 5 for the majority of FENE-CR solutions.

Under Newtonian flow assumptions the shear viscosity is constant, the normal stress difference is zero and there is no elastic contribution. Then, the extensional viscosity is defined as $\eta_{e}=3 \eta_{0}$ and the total stress is expressed as $T=2 \eta_{0} D$ (nb. $\eta_{0}$ is defined above, as the zero-shear-viscosity). Under viscoelastic considerations and the present work, the solvent-viscosity fraction $(\beta)$ is set to 0.9 , which implies a $90 \%$ solvent present to mimic common Boger fluid characteristics (see Aguayo et al. (Aguayo et al. 2008)).

\section{Problem domain and boundary conditions}

The geometrical configurations used and the corresponding meshes are presented in Fig. 2. The configurations differed in degree of contraction ranging from 90 degrees, to 45 and 30 degrees, and down to 15 degrees; yet all share the same contraction ratio of $4: 1$. In addition two hyperbolic configurations have also been 
studied, with the same contraction ratio of $4: 1$, where one configuration has rounded recessive corner and the other a sharp corner. Finite element meshes are composed of triangular elements and detailed mesh characteristics for each configuration is shown in table 2.

The radius of the hyperbolic profile used is described by

$$
r(z)=\frac{r_{0}}{\sqrt{\frac{\mathrm{z}}{\mathrm{H}}\left(r_{0}^{2} / r_{1}^{2}-1\right)+1}}
$$

In the above equation $(E q .7) r_{0}$ is the radius of the die inlet, $r_{1}$ is the radius of the die outlet and $\mathrm{H}$ is the length of the contraction. A hyperbolic contraction is assumed to enable a constant strain rate to be established on the flow centre line throughout the contraction region, and the contribution from shearing there is said to be negligible. A series of three meshes for the hyperbolic contraction, depicted in Fig. 3, has been utilised to establish mesh convergence properties within the corresponding solutions generated. Due to axial symmetry, only half of the flow domain is modeled. The length after the contraction of the 90, 45, 30 and 15 degrees configurations are about 50L allowing a fully developed flow at exit. This is not the case in the hyperbolic contractions, where the geometry ends, somewhat more abruptly, after the contraction to reflect and test the experimental set-up.

No-slip boundary conditions are imposed on the geometry-wall and a symmetry condition is imposed along the centre of the geometry. The problem has thus been solved only for half of the fluid domain. A pressure-driven Poiseuille flow is prescribed at the inlet, and two different exit conditions are imposed at the outlet: a natural streamwise (open) boundary condition and a plug flow. In all cases, a pressure reference is fixed at a single location on flow-exit to remove the indeterminacy of pressure and all solutions are reported under steady-state conditions. 


\section{Pressure-drop}

Under contraction flow, Boger fluids have been shown to substantiate significantly larger pressure drops than those for their equivalent Newtonian fluids (see Nigen \& Walters (Nigen and Walters 2001)). A measure of the pressure drop across a contraction can be expressed by the corrected Excess Pressure Drop (epd) defined as (Aguayo et al. 2008):

$$
e p d=\frac{\left(\Delta p-\Delta p_{f d}\right)_{\mathrm{B}}}{\left(\Delta p-\Delta p_{f d}\right)_{\mathrm{N}}} \text { and } \Delta p_{f d}=\Delta p_{u} L_{u}+\Delta p_{d} L_{d}
$$

The $e p d^{l}$ is thus a fraction of the contribution from a Boger fluid $(B)$ flow divided by the contribution for an equivalent Newtonian fluid $(N)$ flow at the same flow rate, where the $\Delta P$ is the total pressure difference between the inlet and outlet within fully-developed flow, here taken within 1-\% of $\tau, \nabla u$ or $\nabla p$. In this study the point was taken at $1 \%$ of $\tau . \Delta P_{u}, \Delta P_{d}$ represents the fully-developed pressure gradient in the upstream and downstream section respectively and $L_{w}, L_{d}$ are the distances from an inlet sample-point to the start of the contraction and from the start of the contraction to the exit, respectively (Binding et al. 2006).

\section{Numerical solution procedure}

\section{Hybrid finite element/finite volume scheme}

The numerical strategy used in this study to gain an approximate solution to the governing Eq. (1) and (2) is based on semi-implicit Taylor-Galerkin/pressure correction finite element ( $f e$ ) algorithm (TGPC) (Donea 1984) modified by incorporating a sub-cell cell-vertex finite volume $(f v)$ scheme, previously described and utilized in (Wapperom and Webster 1998a), (Webster et al. 2005b). The discretisation is performed based on triangular parent cells consisting of six nodes, three vertex nodes and three mid-side nodes. The velocity $f e$-solutions, located on the vertex and mid-side nodes of the finite element, is approximated with quadratic shape functions. The pressure solution is approximated by linear shape functions and is located only at the vertex nodes. To apply a flux distribution scheme, within the finite volume context only vertex reference is

${ }^{1}$ A relative Couette Correction method 
necessary, for which each parent triangular finite element cell is subdivided into four triangular $f v$-sub-cells.

Concisely, the momentum and continuity equations are solved with a finite element strategy, while the differential constitutive equation of stress is solved with a finite volume strategy (Aboubacar and Webster 2001), both imposed on a time-stepping procedure. The time stepping procedure utilized consists of a twostep Lax-Wenderoff time stepping method based on Taylor series expansion (Donea 1984). Through the pressure splitting and diffusion terms in Eq. (2), the incompressibility constraint is accommodated and second-order accuracy in time is obtained. The tolerance criterion for the time-stepping procedure used in this study is set to $10^{-6}$. On each time step, the numerical scheme transcends three separated stages, as described by Wapperom and Webster (Wapperom and Webster 1999).

At the first stage the momentum and the stress equations are solved and divergence-free velocity fields are calculated given initial velocity and pressure fields. This is obtained by prediction ( $E q .9)$ and then a correction step (Eq. 10). In the following equations $t$ denotes the time step and $n$ the time level, $u u^{*}, p, \tau, d$ the velocity, non-solenoidal velocity, pressure, extra-stress and first rate of strain, respectively. $R e$ and $W e$ are the Reynolds and Weissenberg number defined above.

Stage la (prediction):

$$
\begin{gathered}
\frac{2 \operatorname{Re}}{\Delta t}\left(u^{n+\frac{1}{2}}-u^{n}\right)=[\nabla \tau]^{n}+\nabla \cdot\left(2 \beta \frac{d^{n+\frac{1}{2}}+d^{n}}{2}\right)-\nabla\left(p^{n}+\theta_{1}\left(p^{n}-p^{n-1}\right)\right)+F_{G}^{n} \\
\frac{2 W e}{\Delta t}\left(\tau^{n+\frac{1}{2}}-\tau^{n}\right)=\left(f I-f \tau-W e\left(u \cdot \nabla \tau-L \cdot \tau+\tau \cdot L^{T}\right)\right)^{n}
\end{gathered}
$$

Stage $1 b$ (correction):

$$
\frac{\operatorname{Re}}{\Delta t}\left(u^{*}-u^{n}\right)=[\nabla \tau]^{n+(1 / 2)}+\nabla \cdot\left(2 \beta \frac{d^{*}+d^{n}}{2}\right)-\nabla\left(p^{n}+\theta_{1}\left(p^{n}-p^{n-1}\right)\right)+F_{G}^{n+(1 / 2)},
$$




$$
\frac{W e}{\Delta t}\left(\tau^{n+1}-\tau^{n}\right)=\left(f I-f \tau-W e\left(u \cdot \nabla \tau-L \cdot \tau+\tau \cdot L^{T}\right)\right)^{n+\frac{1}{2}}
$$

At the second stage (Eq. 11), pressure-correction is conducted via a Poisson equation, based on temporal pressure-difference, using the calculated $u^{*}$ from stage 1.

Stage 2:

$$
\nabla^{2}\left(p^{n+1}-p^{n}\right)=\frac{\operatorname{Re}}{\theta_{2} \Delta t} \nabla \cdot\left(u^{*}\right)
$$

In a third stage (Eq. 12), incompressibility is enforced and a divergence-free velocity field $u^{n+1}$ is constructed, appealing the calculated $u^{*}$ and pressuredifference $\left(p^{n+1}-p^{n}\right)$ from stages 1 and 2 .

Stage 3:

$$
\frac{2 \operatorname{Re}}{\Delta t}\left(u^{n+1}-u^{*}\right)=-\theta_{2} \nabla\left(p^{n+1}-p^{n}\right)
$$

A combination of solvers is employed for the different stages in the numerical simulation scheme. For the resulting Galerkin mass matrix-vector equations, formed at stages 1 and 3, an iterative element-by-element Jacobi method is used, while a direct Choleski decomposition solver is invoked for the pressure equation at stage 2 (Hawken et al. 1990).

\section{Finite volume cell vertex scheme}

In the hybrid $f e / f v$ context, we appeal to a cell-vertex finite volume $(f v)$ approach to spatially discretise the extra-stress equations of Stage 1 . This scheme is based upon an upwinding technique (fluctuation distribution), that distributes control volume residuals to provide nodal solution updates. Originally, such upwinding schemes were designed with pure-convection problems in mind. These fluctuation distribution schemes possess beneficial properties such as conservation, linearity preservation and/or positivity (Wapperom and Webster (Wapperom and Webster 1998b)). Concisely, by rewriting the extra-stress equation in non-conservative form, with flux ( $\left.\boldsymbol{R}=\mathbf{u} . \nabla^{\mathbf{T M}}{ }^{\prime}\right)$ and absorbing remaining terms under the source $(Q)$, one may obtain: 


$$
\frac{\partial \tau}{\partial t}+\boldsymbol{R}=\boldsymbol{Q}
$$

We consider each scalar stress component, $\tau$, acting on an arbitrary volume $\Omega=\sum_{l} \Omega_{l}$, whose variation is controlled through corresponding components of fluctuation of the flux $(R)$ and the source term $(Q)$,

$$
\frac{\partial}{\partial t} \int_{\Omega_{l}} \tau d \Omega=-\int_{\Omega_{l}} R d \Omega+\int_{\Omega_{l}} Q d \Omega
$$

The objective is to evaluate the flux and source variations over each finite volume triangle $\left(\Omega_{l}\right)$, with their distribution to its three vertices according to the preferred strategy. The resulting nodal update for a particular node $(l)$ is obtained by accumulating the contributions from its control volume $\Omega_{l}$, composed of all $f v$ triangles surrounding node $(l)$. The flux and source residuals may be evaluated over different control volumes associated with a given node $(l)$ within the $f v$-cell $\mathrm{T}$; namely, the contribution governed over the fv-triangle $\mathrm{T},\left(R_{T}, Q_{T}\right)$, and that subtended over the median-dual-cell zone ${ }^{2},\left(R_{m d c}, Q_{m d c}\right)$. This procedure demands appropriate area-weighting to maintain consistency, which for temporal accuracy has been extended to time-terms likewise. With the candidate stress equation considered as split into time derivative, flux and source, and integrated over associated control volumes, the concise generalized fv-nodal update equation may be expressed per stress component as,

$$
\left[\sum_{\forall T_{l}} \delta_{T} \alpha_{l}^{T} \Omega_{T}+\sum_{\forall M D C_{l}}\left(1-\delta_{T}\right) \ddot{\Omega}_{l}^{T}\right] \frac{\Delta \tau_{l}^{n+1}}{\Delta t}=\sum_{\forall T_{l}} \delta_{T} \alpha_{l}^{T} b^{T}+\sum_{\forall M D C_{l}}\left(1-\delta_{T}\right) b_{l}^{M D C}
$$

where $b^{T}=\left(-R_{T}+Q_{T}\right), b_{l}^{M D C}=\left(-R_{M D C}+Q_{M D C}\right)^{l}$. Here, $\Omega_{T}$ is the area of the $f v$ triangle $T$, whilst $\hat{\Omega}_{l}^{T}$ is that of its median-dual-cell $(M D C)$. Parameter $\delta_{T}$ directs the balance taken between the contributions from the median-dual-cell and the $f v$ triangle $T$, with $0 \leq \delta_{T} \leq 1$, see Webster et al. (Webster et al. 2005a). This

\footnotetext{
${ }^{2}$ See (Wapperom and Webster 1999)
} 
expression recognizes segregated fluctuation distribution and median dual cell contributions, area weighting and upwinding factors ( $\alpha_{l}^{T}$-scheme dependent).

\section{Results and discussion}

\section{Mesh refinement}

Specifically, all the following solutions presented in the results section are expressed in a non-dimensional form. Firstly, to explore solution mesh convergence, simulations of increasing levels of mesh densities (table 2) are performed for both Newtonian and Boger fluid flow (Fig. 3). The velocity and strain rate solutions are analysed along the symmetry line in the flow direction. The velocity in the flow direction (z-direction) is depicted in Fig. 4a., for three mesh densities. The velocity is constant until the start of the contracting region $(\mathrm{z}=0)$, from which point it increases linearly. The strain rate in the flow direction is depicted in Fig. 4b., showing a considerable increase at the start of the contracting region and a relatively constant strain rate throughout the contraction. The solutions computed from the various meshes lie in close agreement, with no pronounced difference (less than 1\%) being observed between the results for the coarse, medium and refined mesh. Thus the coarse-medium meshes have been used as the basis for further evaluation due to their efficiency in calculation time.

\section{Exit conditions}

Two different exit conditions have been trialled on the hyperbolic contraction configurations. In the one case, the boundary exit remained free (natural condition) and in the other case the exit boundary condition was set to be a jetplug flow with the velocity set to unity. Solutions for both Newtonian and Boger fluids were studied at $W e$-numbers ranging from 0.1 to 50 . The strain rates from the different exit conditions are at the same level but an oscillation can be seen near the exit, especially in the FENE-CR solutions when plug flow exit boundary conditions apply; this is due to the flow adjustment there, anticipating a jet outlet. The impact of the exit conditions in the solution along the wall was also analysed and there is no significant difference in shear rate noted between the different exit conditions. Once again, an oscillation is observed, especially for the plug-type boundary condition. Note, however, that this oscillation is a highly localised 
effect, and does not disturb the solution in the domain; hence, the flow at exit may be ignored. The natural exit condition was used in the subsequent simulations performed in this study, due to the less pronounced oscillation.

\section{Vortex formation in different geometries}

The stream traces of the different geometries have also been studied to identify whether any vortices are formed in the fluid domain. Both Newtonian and viscoelastic Boger fluids were studied for all the contraction configurations for Weissenberg numbers ranging from $W e=0.1$ up to $W e=100$. From these simulations it can be seen that the only vortex formed is in the corner of the 90degree contraction for both Newtonian and Boger fluids, increasing with higher flow rate or elasticity. The vortex is already developing at $W e=0.1$ and is depicted in Fig. 5a. No vortices are formed in the other geometries, even for higher We- or $R e$-numbers, see Fig. 5b, as illustrated in stream traces of the hyperbolic contraction at the extreme elasticity level of $W e=100$. No vortices are formed because of the smoothed hyperbolic shape of the contraction and the relatively low contraction ratio. This can be compared with the experimental work of Nguyen, where vortices where formed for 90-degree and 60-degree contractions but not for 30-degree contractions (Nguyen and Boger 1984). Thus, stable steady flow is shown to be established for all geometries (in a reasonable velocity range).

\section{Shape of the Corner}

The impact of the shape of the corner has also been evaluated for the hyperbolic contraction configuration. Numerical simulations have been performed for hyperbolic contractions, one with a rounded corner and one with a sharp corner. Previous studies of 90-degree contractions have shown that a rounded corner delays the development of vortices to higher Weissenberg numbers as compared to a sharp corner (Rothstein and McKinley 2001). The viscoelastic FENE-CR solutions for the hyperbolic contraction can be seen in Fig. 6a-b., which plots the strain rate along the symmetry line at $L=5, W e=1,5$ and 10 . The strain rate along the symmetry line is rising sharply at the start of the contraction $(z=0)$ and finally reaches a relatively constant value of about 0.12 units. The strain rate is seemingly unaffected by the shape of the corner, sharp or rounded, except around $z=5$ where a minimal difference in (0.0006 units) can be observed. 
The behaviour of the normal stress difference $\left(N_{l}\right)$ for increasing We -numbers is studied both along the symmetry line (in extension) Fig. 7a. and along the wall (in shear) Fig. $7 b$. in both geometries. There is a rise in $N_{l}$ along the symmetry line for both corner types in Fig.7a. and a slightly higher $N_{l}(0.007$ units $)$ can be observed for the sharp corner compared to the rounded, indicating a larger stress in the flow direction for the sharp corner instance. The difference in $N_{l}$ is more pronounced with rising $W e$-numbers. Examining $N_{l}$ along the wall in Fig. 7b., a rise in $N_{l}$ throughout the whole contraction is observed, but there is no significant difference between sharp and round corner at increasing $W e$-numbers.

The shear rate along the wall for round and sharp corner is shown in Fig. 8. The shear rate rises sharply at the start of the contraction region to a relatively constant value of around 1.13 units. The influence of the corner shape on the solution is minor and thus the sharp corner option will be retained for future comparison with the experimental set-up.

\section{Flow behaviour in different geometries}

The predicted solutions of the dimensionless strain rate for viscoelastic Boger fluid $(L=5, W e=5)$ in different configurations are displayed through contour plots of the contraction domain in Fig. 9a-f. The appearance of the strain rate for both Newtonian and Boger fluids resembles each other. The area of high extension is concentrated around the regions of the contractions and increases for lower contraction angles. An inhomogeneous uniaxial extension along the flow centreline arises for the 90 to 15-degree contractions, while it is dispersed throughout the whole contraction for both hyperbolic contractions. Line graphs extracted from the contour plots of the strain rate for viscoelastic FENE-CR model $(L=5, W e=5)$ along the symmetry line are displayed in Fig. 10a. The start of the contraction is indicated by a dashed line. Peaks at the contraction are observed for all configurations, with the exception of the hyperbolic contraction case, where the strain rate reaches a nearly constant value of 0.13 units throughout the contracting domain. The 45-degree configuration displays a higher peak (1.24 units) than the 90-degree one (1.02 units), but there is generally a smoothing of the curves and a decrease in the maximum strain rate moving from higher to lower contraction angles. The reason for the peak increase in the 45-degree contraction, 
compared to the 90-degree contraction, is the vortex formation observed in the 90degree configuration (Fig. 5a).

The impact of the first normal stress difference $\left(N_{l}\right)$ along the symmetry line for the different configurations is seen in Fig. 10b. The curves of $N_{l}$ are observed to follow the trends in strain rate for all configurations except that of the hyperbolic contraction, where the level of $N_{l}$ increases throughout the contraction geometry towards the exit. This is due to the fact that there is a small increase in strain rate towards the exit of the hyperbolic contractions, which will be more pronounced in the first normal stress difference $N_{l}$. A further observation taken from Fig. 10 is that the peaks are shifted away from the start of the contraction plane with decreasing contraction angles. This is thought to be due to the sharper contraction taking place at the end of the contractions for the 45-degree, 30-degree and 15degree angle contractions.

The variation of pressure along the symmetry line for a Newtonian fluid in the different configurations is presented in Fig 11. The flow is allowed to fully develop after the contraction in the 90-degree, 45-degree, 30-degree and 15degree contraction configurations. This is not the case for the hyperbolic contraction geometry, where the flow is interrupted immediately after the contraction, to mimic the experimental setup. The maximum pressure is somewhat similar and higher for the 45-degree, 30-degree and 15-degree contractions (around 560 units), than for the 90-degree contraction (400 units), again due to corner vortex formation. A low maximum pressure is observed for the hyperbolic contraction (20 units) and the pressure drop is smoothed around the contraction region as compared to the other configurations. Since the contracting region in the hyperbolic geometry is smoother, the force required to push the fluid through the contraction is necessarily lower, which in turn yields a lower pressure drop over the geometry. Higher forces can thus be used which will lead to a broader measuring range, enabling higher strains and strain rates. The vortex in the 90 degree contraction contributes to a flow field that resembles the hyperbolic contraction configurations, eliminating the sharp corners and, thus reducing the pressure drop. 
The excess pressure drop $(e p d)$ data at different We-numbers in the case of the 90degree contraction and the hyperbolic contraction is depicted in Fig. 12a-b., respectively. Solutions are shown for three increasing $L$-parameters $(L=3,5$ and10). Recall, that excess pressure drop is a corrected measure for pressure drop across the contraction, defined above in (Eq. 8). Previously, (epd)-data has been studied extensively for the 90-degree contraction (4:1 and 4:1:4), showing both increases and decreases in epd with increasing We-numbers, dependent on geometry, solvent fraction and model choice -Oldroyd-B and FENE-CR models (Aguayo et al. 2008; H. R. Tamaddon-Jahromi 2010). Nevertheless, any epd increase derived for FENE-CR $(\beta=0.9, \mathrm{~L}=5)$ in $4: 1: 4$ contractions proved to be only modest (est. $28 \%$ up to $W e<70$ ). In comparison and within the present study, notably pronounced increase in $e p d$ has been demonstrated with FENE-CR $(\beta=0.9, L=5)$ at $W e<50$ for both contraction geometries 90-degree and hyperbolic. For the 90-degree contraction data, epd results initially lie below the unitNewtonian reference line for $W e<10$ (argued to be due to $N_{l}$ dominance over extensional viscosity; see (Nigen and Walters 2001), (H. R. Tamaddon-Jahromi 2010).

In contrast, the hyperbolic contraction displays epd-data larger than unity for all $W e$-numbers -adding greater weight to confirm the successful suppression of $N_{1^{-}}$ influence in this geometry choice. Upon increasing the extensibility parameter $(L)$, epd-levels naturally rise: achieving impressively large relative increases of (24\%, $54 \%, 160 \%$ ); for $\mathrm{L}=3,5,10$. These results correlate well with the experimental results of Nigen and Walters (Nigen and Walters 2001), who reported significant enhancement in pressure drop over a Newtonian fluid for viscoelastic Boger fluids and a 90-degree contraction, as did McKinley and Rothstein (Rothstein and McKinley 1999) likewise for the 4:1:4 geometry. Moreover, McKinley and Rothstein (Rothstein and McKinley 2001) studied the influence of the contractionexpansion ratio and found a generally enhanced pressure drop above that of a Newtonian fluid for increasing $W e$-numbers, indicating a correlation between the pressure drop and strain rate for viscoelastic fluids. Thus, one may conclude that it is indeed a practical proposition to determine extensional fluid properties in the hyperbolic contraction geometry, based upon the sizable quantities that have been shown to be generated. 
The numerical predictions in this study show that the shear rate is minimized and a nearly constant deformation is achieved along the flow centreline for the hyperbolic geometry-shape, as assumed in the work by Wikström and Bohlin (Wikström and Bohlin 1999a), notably in contrast to that generated in the other geometries tested. Due to the nearly constant extensional flow throughout the hyperbolic contraction configuration, this guarantee that test samples will be stretched for a longer period of time, which in turn facilitates practical determination of the extensional viscosity of the sample. The extensional viscosity can be determined from the first normal stress difference in extension $\left(N_{1}=\tau_{z z}-\tau_{r r}\right)$ and the strain rate as follows: $\eta_{e}=N_{1} / d U_{z} d z$. The extensional viscosity thus reflects the strain rate, which is relatively constant on the centreline throughout the entire nozzle-length in the hyperbolic configuration. These results are consistent with earlier experimental findings that used Ultrasound Velocity Profiling (UVP) on hyperbolic contractions (Zatti et al. 2009).

Moreover, such numerical predicted solutions may be compared to corresponding theoretical rheometric functions (see Fig. 13a-extensional viscosity $\eta_{e}$, and Fig. $13 \mathrm{~b}$-first normal stress difference $N_{l}$ ), which establishes the deformation ranges of agreement and the proximity to steady pure extensional flow. The predicted solutions are plotted against localised Weissenberg Number, determined at the steady deformation rate reached at a sample-point $(z=9)$ along the flow centreline and on the wall for prediction of $\eta_{e}$ and $N_{l}$, respectively. The centreline extensional data findings are promising, in that there is close agreement established at all three L-values, $(L=3,5,10)$, on limiting $\eta_{e}$ plateaux reached at $W e \geq 10$; trends agree well across a wide range of $W e$ with $\mathrm{L}=3$; yet show a degree of departure during intermediate ranges of $0.3 \leq W e \leq 5$ for $L=5$ and $0.3 \leq W e \leq 10$ for $L=10$. Finally on shear data, the first normal stress difference results on the wall, shown in Fig 13b, are even more encouraging. The numerical predictions agree well with the theoretical results for $\mathrm{We} \geq 1$ and for all values tested of the extensibility parameter, $\mathrm{L}=(3,5,10)$. Clearly, there is some $N_{l}$ influence in this flow. Here, one may note the Szabo et al. (Szabo et al. 1997) argument re dependency upon the $\operatorname{FENE}(L)$-parameter under a highly polymeric state, in that a trade-off applied between using small finite extensions $(L)$ that produce smaller 
extensional viscosities, but require less stretch to attain the extension $(L)$, and hence become effective at lower Deborah numbers. This would appear to be the case here. Accordingly, this procedure also allows a functional relationship to be established between epd and extensional viscosity ${ }^{3},-$ one of our original targets, so eagerly sought after under experimental practice.

\section{Conclusion}

Various simulations have been performed in this work of contraction flows of Newtonian and viscoelastic Boger fluids. This covers different contraction geometries in an attempt to optimize on geometry selection with respect to extraction of constant extensional flow. Numerical computations of steady-state Boger fluid flow through various configurations have been analysed using the FENE-CR constitutive model. Stream traces of the flow reveal corner vortex development in the 90-degree contraction; and yet no vortices for the 45-degree, 30-degree and 15-degree contraction options, nor for the hyperbolic contraction. Two different exit conditions were imposed on the hyperbolic contraction flow, and the influence of the exit conditions are seen to apply locally, only around the exit itself; hence, do not affect findings otherwise.

In addition, the influence on the extensional deformation generated by the sharp or rounded recess corner of the hyperbolic contraction has been shown to be minor for a contraction ratio of 4:1. Nevertheless, with future experimental design firmly in mind, a contraction with a round corner would serve to further minimize shear stress contributions, especially at higher contraction ratios. The pressure drop, strain rate and extensional viscosity through the various contraction variants reveal that the hyperbolic contraction is the one that is best suited for the generation of constant extension in the measuring system and in minimizing shear contribution at the flow centre-line. Theoretical to predicted solution correspondence has been established and leads to new means to practically measure extensional properties, linking these to pressure drops. The hyperbolic contraction flow exposes the fluid to a longer time period of deformation as compared to other contraction flow instances. This is a highly desirable property

\footnotetext{
${ }^{3}$ See Cogswell analysis in Rothstein \& McKinley paper (Rothstein and McKinley 1999)
} 
in a measuring system for extensional flow and also indicates that the use of a hyperbolic contraction in pipe contractions will generate a more uniform flow.

To resemble the numerical problem to the experimental set-up it would be illuminating to introduce a die swell exit condition on the hyperbolic configurations in a future study. It would also be of interest to analyse the flow with slip imposed on the die walls to further minimize the effect of shear stress on the sample and determine the properties of the flow for shear-thinning fluids through the hyperbolical die. This would prove particularly illuminating in attempting to segregate the various conflicting contributions to pressure-drops, namely extensional viscosity and first normal stress difference in shear.

\section{References}

Aboubacar M, Webster MF (2001) A cell-vertex finite volume/element method on triangles for abrupt contraction viscoelastic flows. Journal of Non-Newtonian Fluid Mechanics 98 (2-3):83-106

Aguayo JP, Tamaddon-Jahromi HR, Webster MF (2008) Excess pressure-drop estimation in contraction and expansion flows for constant shear-viscosity, extension strain-hardening fluids. Journal of Non-Newtonian Fluid Mechanics 153 (2-3):157-176. doi:10.1016/j.jnnfm.2008.05.004

Baird DG, Huang J (2006) Elongational Viscosity Measurements Using a Semi-Hyperbolic Die. Appl Rheol $16(6): 312-320$

Baird DGC, T W. McGrady, C. Mazahir, S M. (2010) Evaluation of the Use of a Semi-hyperbolic Die for Measuring Elongational Viscosity of Polymer Melts. Appl Rheol 20:34900-34901 - 34900-34912

Binding DM (1988) An approximate analysis for contraction and converging flows. J Non Newt Fluid Mech 27:173-189

Binding DM, Couch MA, Walters K (1998) The pressure dependence of the shear and elongational properties of polymer melts. Journal of Non-Newtonian Fluid Mechanics 79 (2-3):137-155

Binding DM, Phillips PM, Phillips TN (2006) Contraction/expansion flows: The pressure drop and related issues. J Non-Newton Fluid Mech 137 (1-3):31-38. doi:10.1016/j.jnnfm.2006.03.006

Boger DV (1987) Viscoelastic flows through contractions. Annu Rev Fluid Mech 19:157-182

Chilcott MD, Rallison JM (1988) CREEPING FLOW OF DILUTE POLYMER-SOLUTIONS PAST

CYLINDERS AND SPHERES. Journal of Non-Newtonian Fluid Mechanics 29 (1-3):381-432

Collier JR, Romanoschi O, Petrovan S (1998) Elongational rheology of polymer melts and solutions. Journal of Applied Polymer Science 69 (12):2357-2367

Donea J (1984) A TAYLOR-GALERKIN METHOD FOR CONVECTIVE-TRANSPORT PROBLEMS. Int J Numer Methods Eng 20 (1):101-119

Entov VM, Hinch EJ (1997) Effect of a spectrum of relaxation times on the capillary thinning of a filament of elastic liquid. Journal of Non-Newtonian Fluid Mechanics 72 (1):31-53

Fuller GG, Cathey CA, Hubbard B, Zebrowski BE (1985) Extensional viscosity measurements for lowviscosity fluids. J Rheol 31:235-249

H. R. Tamaddon-Jahromi MFW, K. Walters (2010) Predicting numerically the large increases in extra pressure drop when boger fluids flow through axisymmetric contractions. Natural Science 2 (1):1-11 
Hawken DM, Tamaddonjahromi HR, Townsend P, Webster MF (1990) A TAYLOR-GALERKIN-BASED ALGORITHM FOR VISCOUS INCOMPRESSIBLE-FLOW. Int J Numer Methods Fluids 10 (3):327-351 James DF, Chandler GM, Armour SJ (1990) A CONVERGING CHANNEL RHEOMETER FOR THE MEASUREMENT OF EXTENSIONAL VISCOSITY. Journal of Non-Newtonian Fluid Mechanics 35 (23):421-443

Kim HC, Pendse A, Collier JR (1994) POLYMER MELT LUBRICATED ELONGATIONAL FLOW. Journal of Rheology 38 (4):831-845

Meissner J (1972) Development of a universal extensional rheometer for the uniaxial extension of polymer melts. Trans Soc Rheol 16:405-420

Meissner J, Hostettler J (1994) A NEW ELONGATIONAL RHEOMETER FOR POLYMER MELTS AND OTHER HIGHLY VISCOELASTIC LIQUIDS. Rheologica Acta 33 (1):1-21

Nguyen TH, Boger DV (1984) THE INFLUENCE OF ELASTICITY ON DIE ENTRY FLOWS. Journal of Rheology 28 (5):654-654

Nigen S, Walters K (2001) On the two-dimensional splashing experiment for Newtonian and slightly elastic liquids. J Non-Newton Fluid Mech 97 (2-3):233-250. doi:10.1016/s0377-0257(00)00221-4

Oom A, Pettersson A, Taylor JRN, Stading M (2008) Rheological properties of kafirin and zein prolamins. Journal of Cereal Science 47 (1):109-116

Rothstein JP, McKinley GH (1999) Extensional flow of a polystyrene Boger fluid through a $4: 1: 4$ axisymmetric contraction/expansion. Journal of Non-Newtonian Fluid Mechanics 86 (1-2):61-88

Rothstein JP, McKinley GH (2001) The axisymmetric contraction-expansion: the role of extensional rheology on vortex growth dynamics and the enhanced pressure drop. J Non-Newton Fluid Mech 98 (1):33-63

Sridhar T, Tirtaatmadja V, Nguyen DA, Gupta RK (1991) Measurement of Extensional Viscosity of PolymerSolutions. Journal of Non-Newtonian Fluid Mechanics 40 (3):271-280

Stading M, Bohlin L (2000) Measurements of extensional flow properties of semi-solid foods in contraction flow. Proceedings of the 2nd International Symposium on Food Rheology and Structure 2:117-120

Stading M, Bohlin L (2001) Contraction flow measurements of extensional properties. Transactions of the Nordic Rheology Scociety 8/9:147-150

Szabo P, Rallison JM, Hinch EJ (1997) Start-up of flow of a FENE-fluid through a 4:1:4 constriction in a tube. J Non-Newton Fluid Mech 72 (1):73-86. doi:10.1016/s0377-0257(97)00023-2

Walters K, Webster MF (2003) The distinctive CFD challenges of computational rheology. Int J Numer Methods Fluids 43 (5):577-596. doi:10.1002/fld.522

Wapperom P, Webster MF (1998a) A second-order hybrid finite-element/volume method for viscoelastic flows. Journal of Non-Newtonian Fluid Mechanics 79 (2-3):405-431

Wapperom P, Webster MF (1998b) A second-order hybrid finite-element/volume method for viscoelastic flows. J Non-Newtonian Fluid Mech 79:405-431

Wapperom P, Webster MF (1999) Simulation for viscoelastic flow by a finite volume/element method. Comput Meth Appl Mech Eng 180 (3-4):281-304

Webster MF, Matallah H, Sujatha KS (2005a) Sub-cell approximations for viscoelastic flows-filament stretching. J Non-Newtonian Fluid Mech 126:187-205

Webster MF, Tamaddon-Jahromi HR, Aboubacar M (2005b) Time-dependent algorithms for viscoelastic flow: Finite element/volume schemes. Numer Meth Part Differ Equ 21 (2):272-296. doi:10.1002/num.20037 White SA, Gotsis AD, Baird DG (1987) REVIEW OF THE ENTRY FLOW PROBLEM - EXPERIMENTAL AND NUMERICAL. Journal of Non-Newtonian Fluid Mechanics 24 (2):121-160

Wikström K, Bohlin L (1999a) Extensional flow studies of wheat flour dough. I. Experimental method for measurements in contraction flow geometry and application to flours varying in breadmaking performance. Journal of Cereal Science 29 (3):217-226 
Zatti D, Wiklund J, Vignali G, Stading M (2009) Determination of Velocities Profiles in Hyperbolic Contraction using Ultrasound Velocity Profiling. Annual Transactions of the Nordic Rheology Society 17:277-280

\section{Figures}

\section{Figure 1}
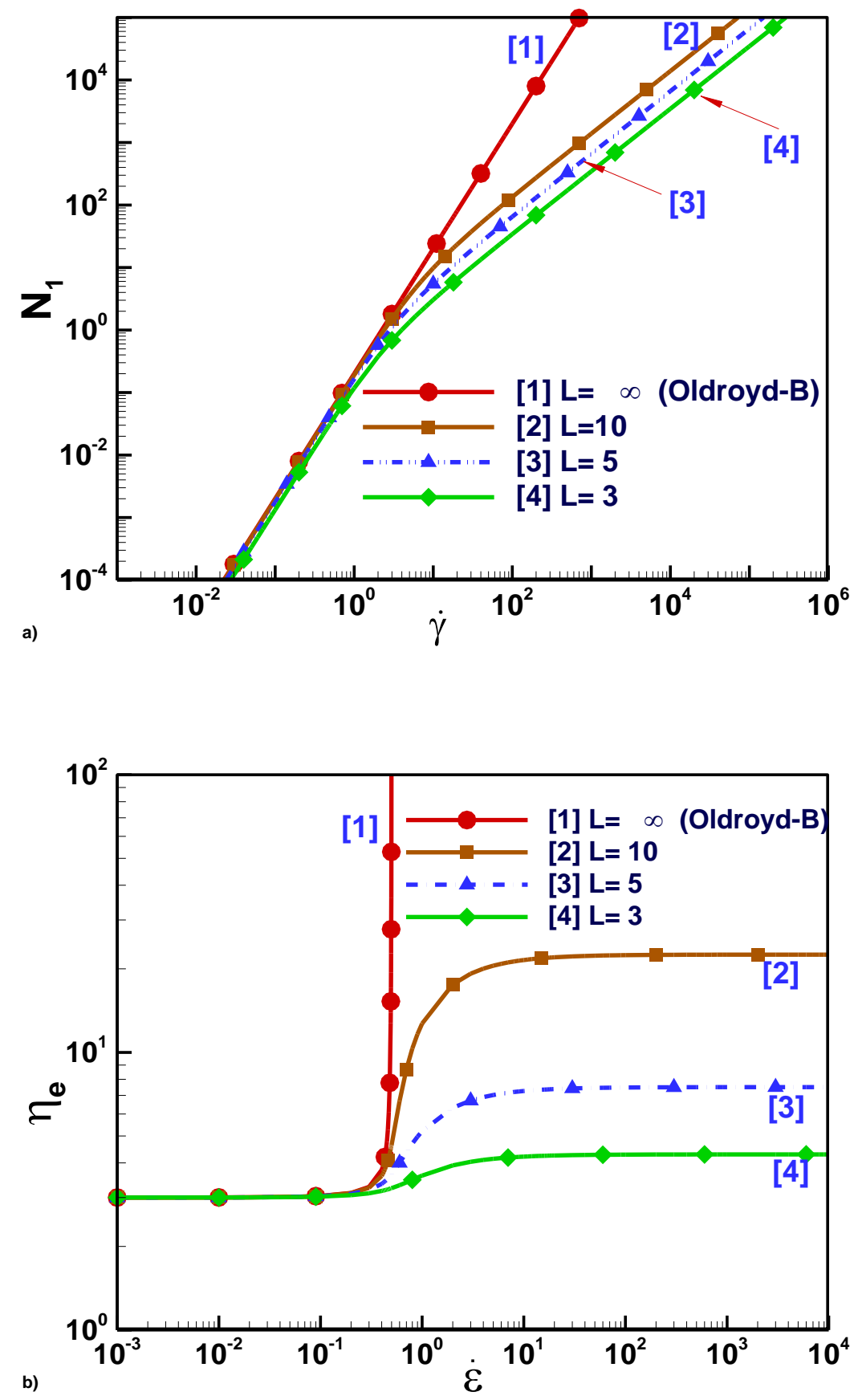


\section{Figure 2}
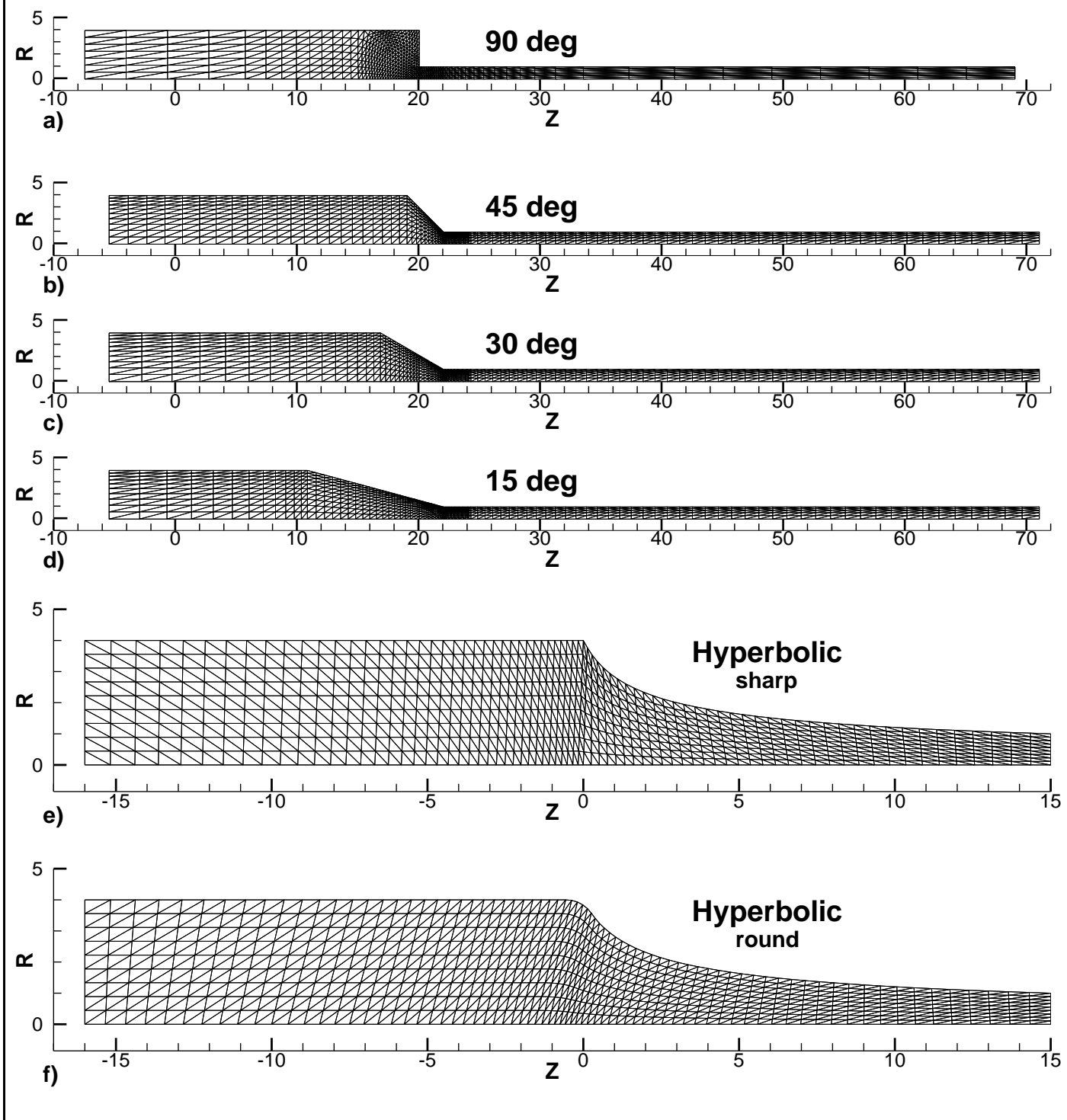
Figure 3

Mesh A

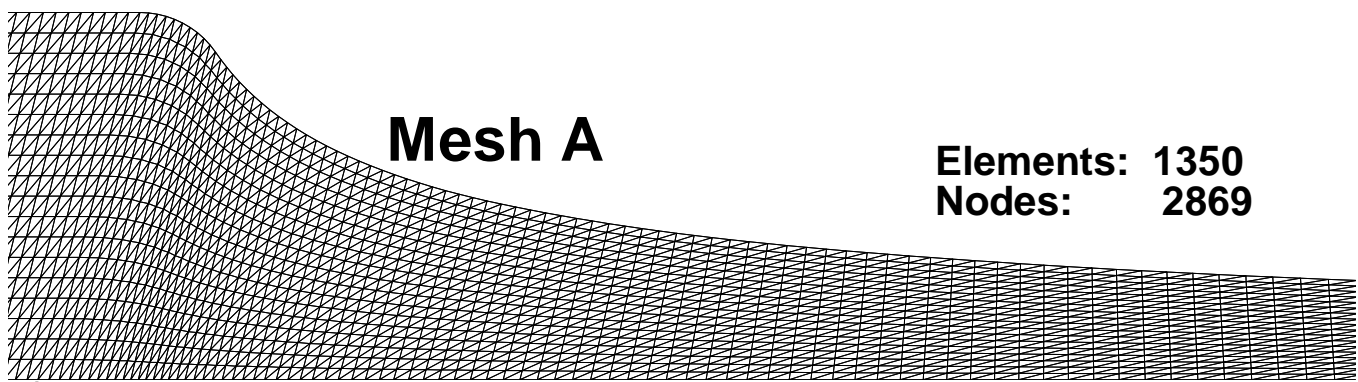

a)

Mesh B

Nodes: $\quad 3591$

Mesh C

Elements: 2160

Nodes: $\quad 4525$

c) 
Figure 4
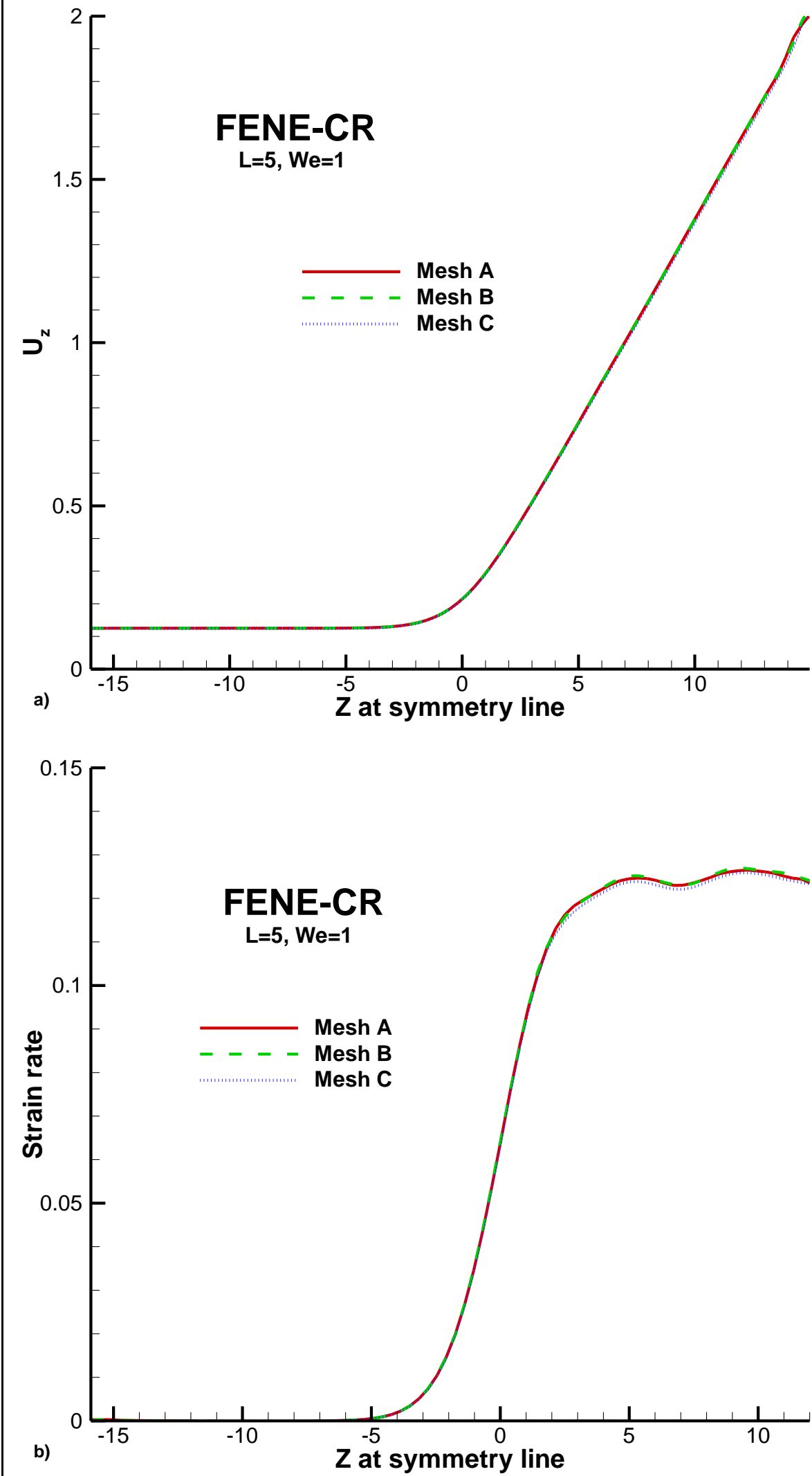
Figure 5

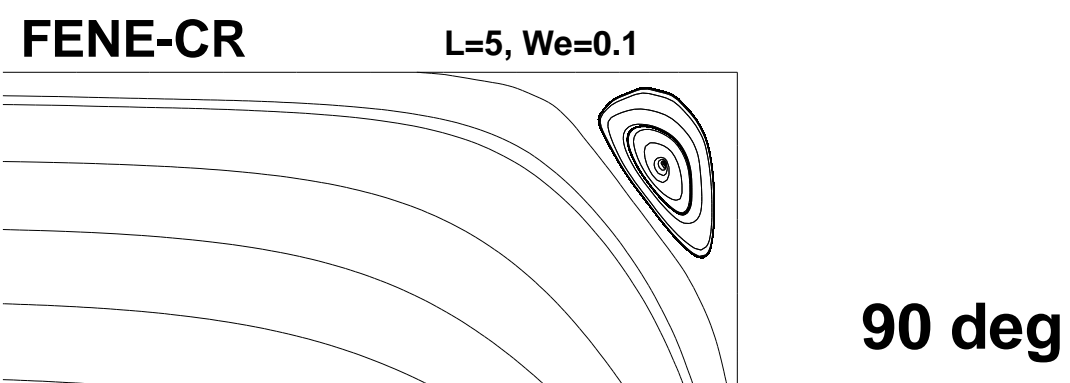

a)

FENE-CR $L=5, W e=100$

b)

Hyperbolic

sharp corner

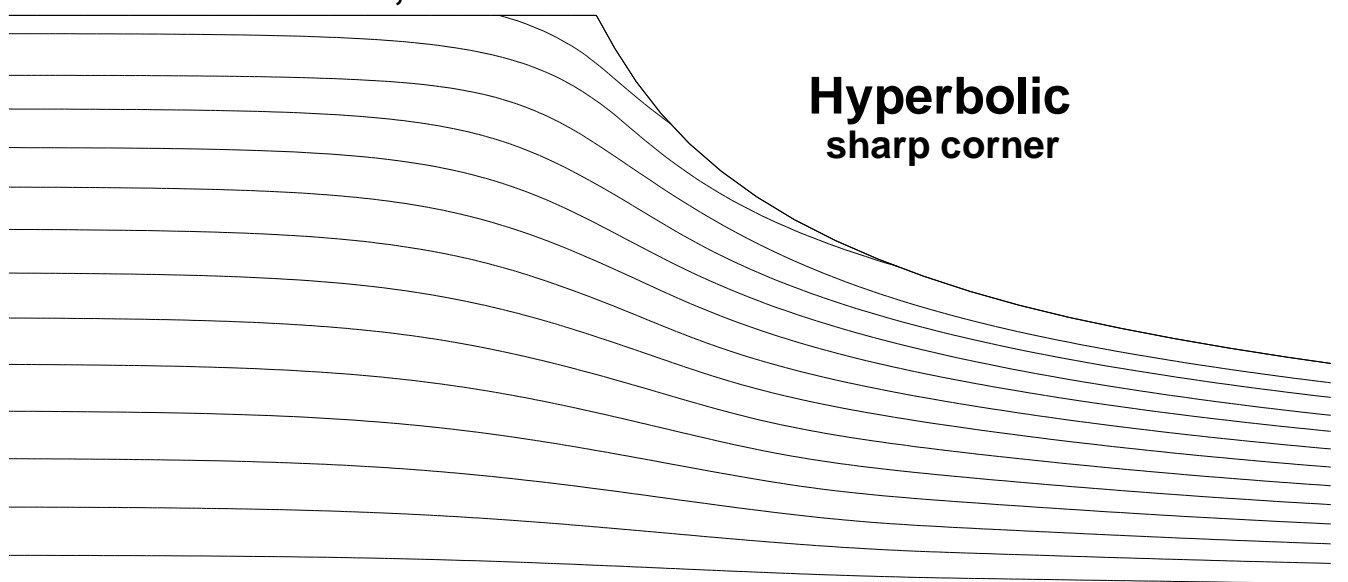




\section{Figure 6}
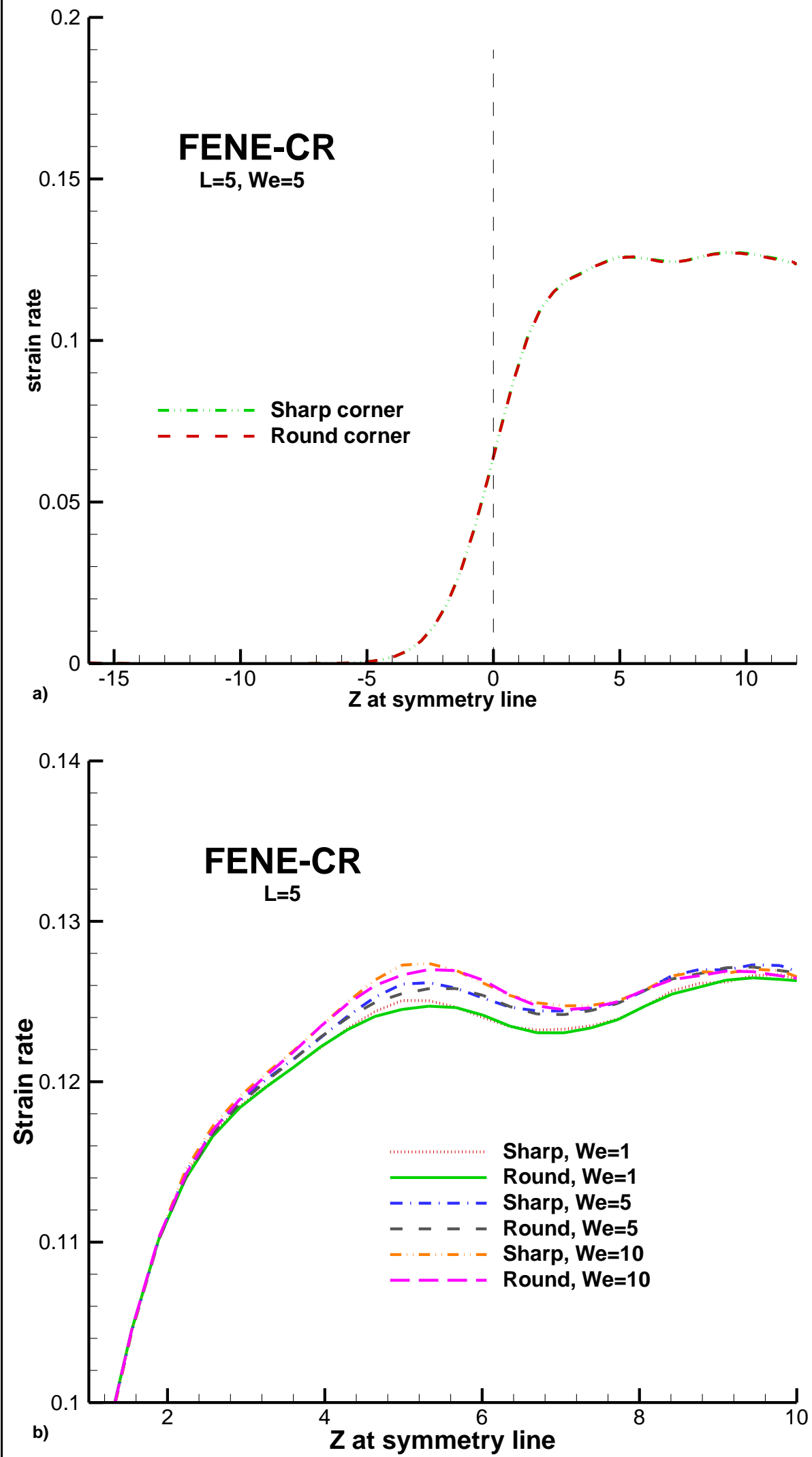


\section{Figure 7}
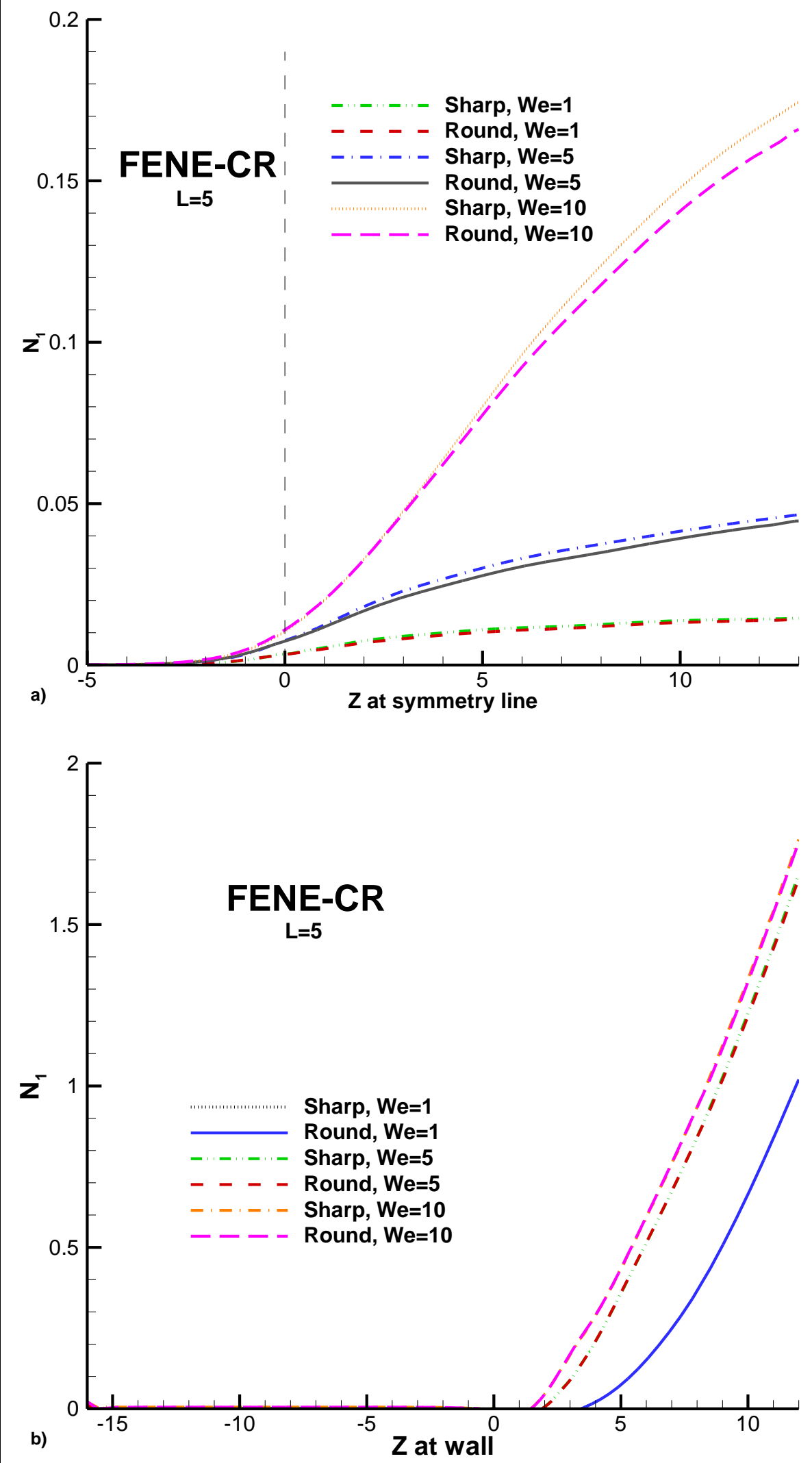
Figure 8

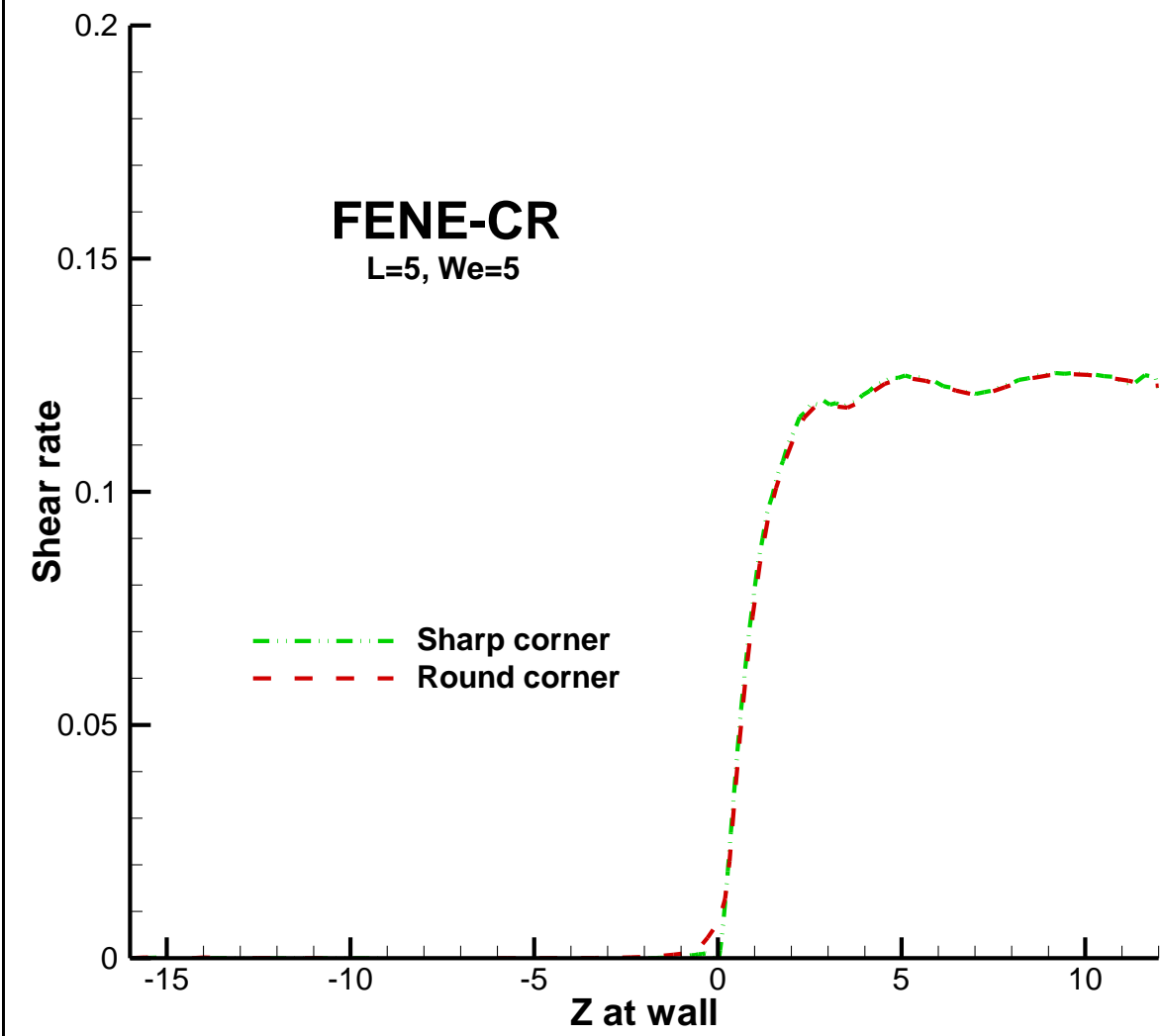




\section{Figure 9}

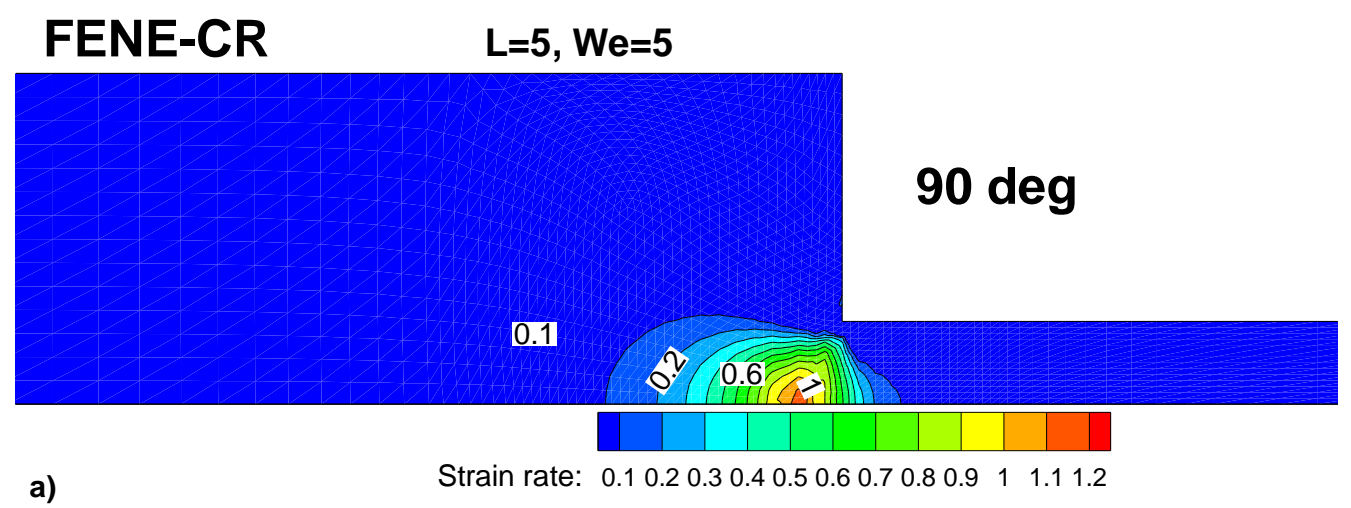

\section{FENE-CR $\quad \mathrm{L}=5, \mathrm{We}=5$}

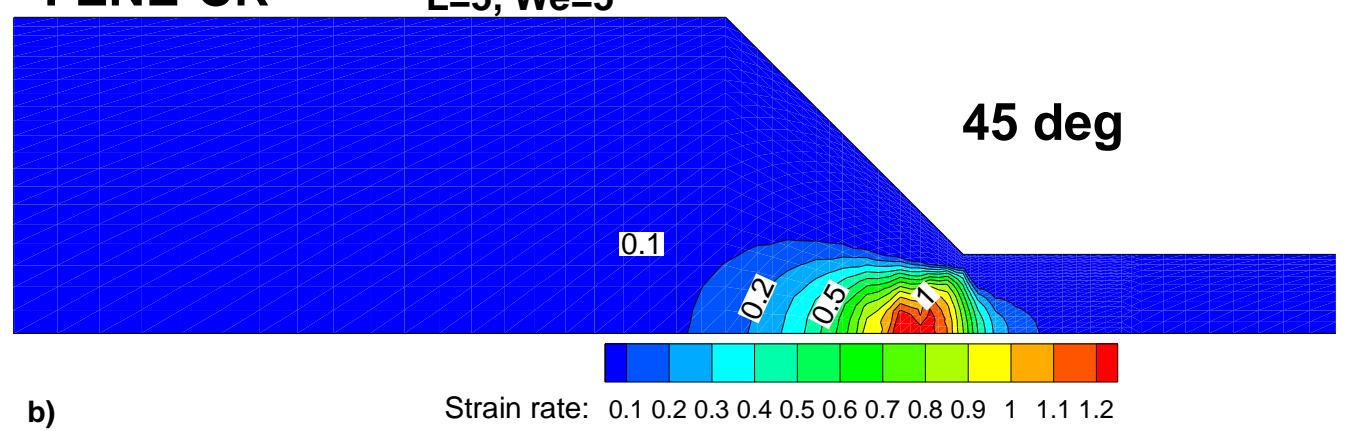

\section{FENE-CR $\quad \mathrm{L}=5, \mathrm{We}=5$}

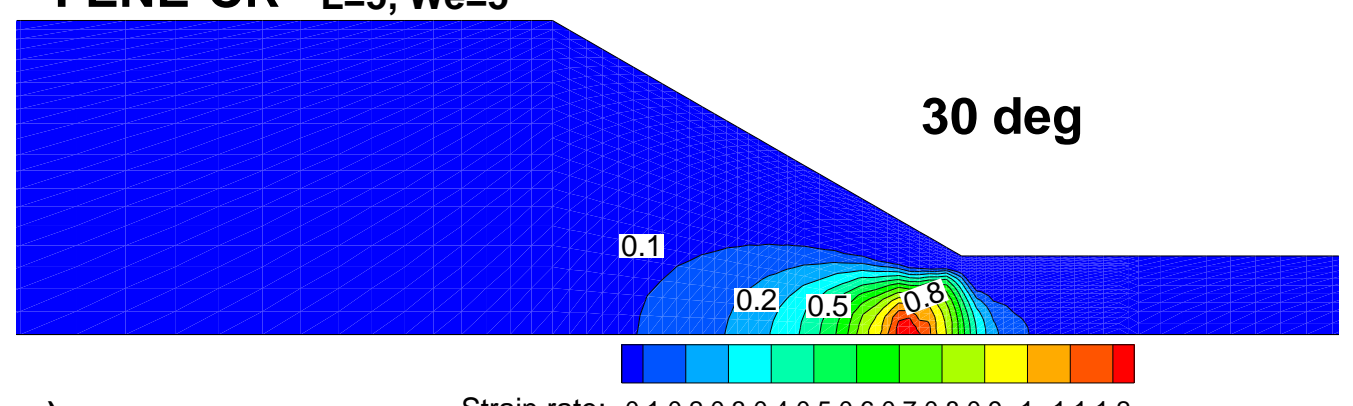

c)

Strain rate: 0.10 .20 .30 .40 .50 .60 .70 .80 .9111 .11 .2

\section{FENE-CR $L=5, W e=5$}

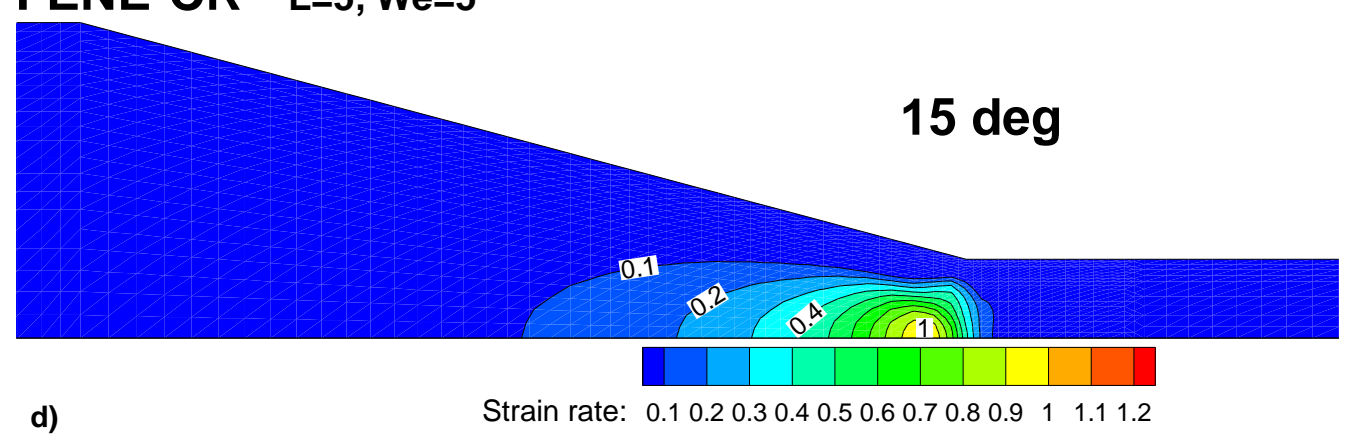


FENE-CR L $=5$, We $=5$

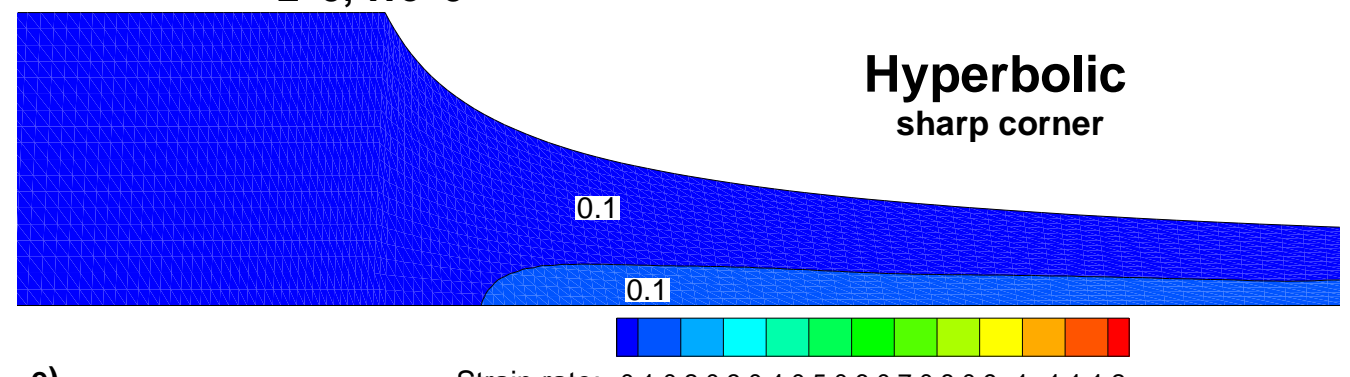

e)

Strain rate: $\quad 0.10 .20 .30 .40 .50 .60 .70 .80 .9 \quad 11.11 .2$

\section{FENE-CR $\quad \mathrm{L}=5, \mathrm{We}=5$}

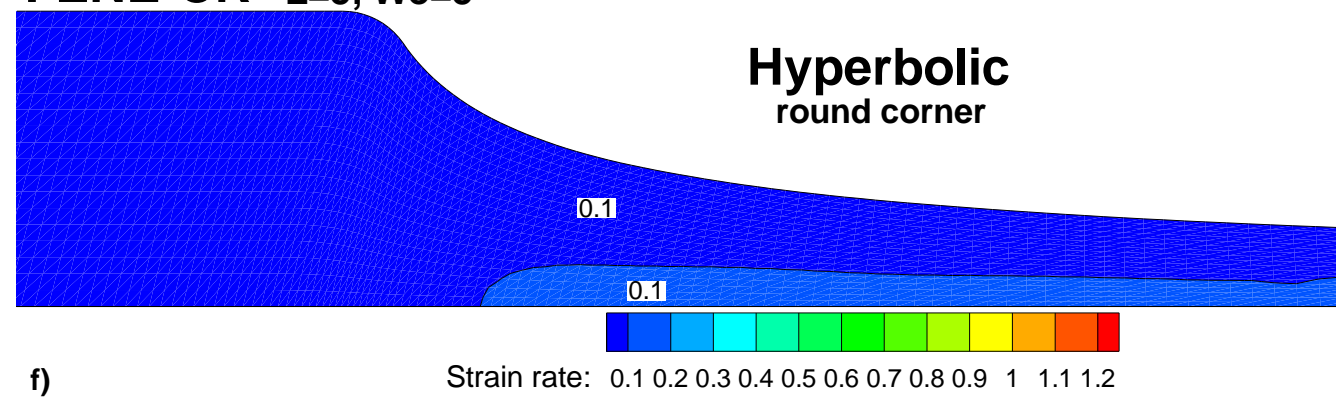


Figure 10
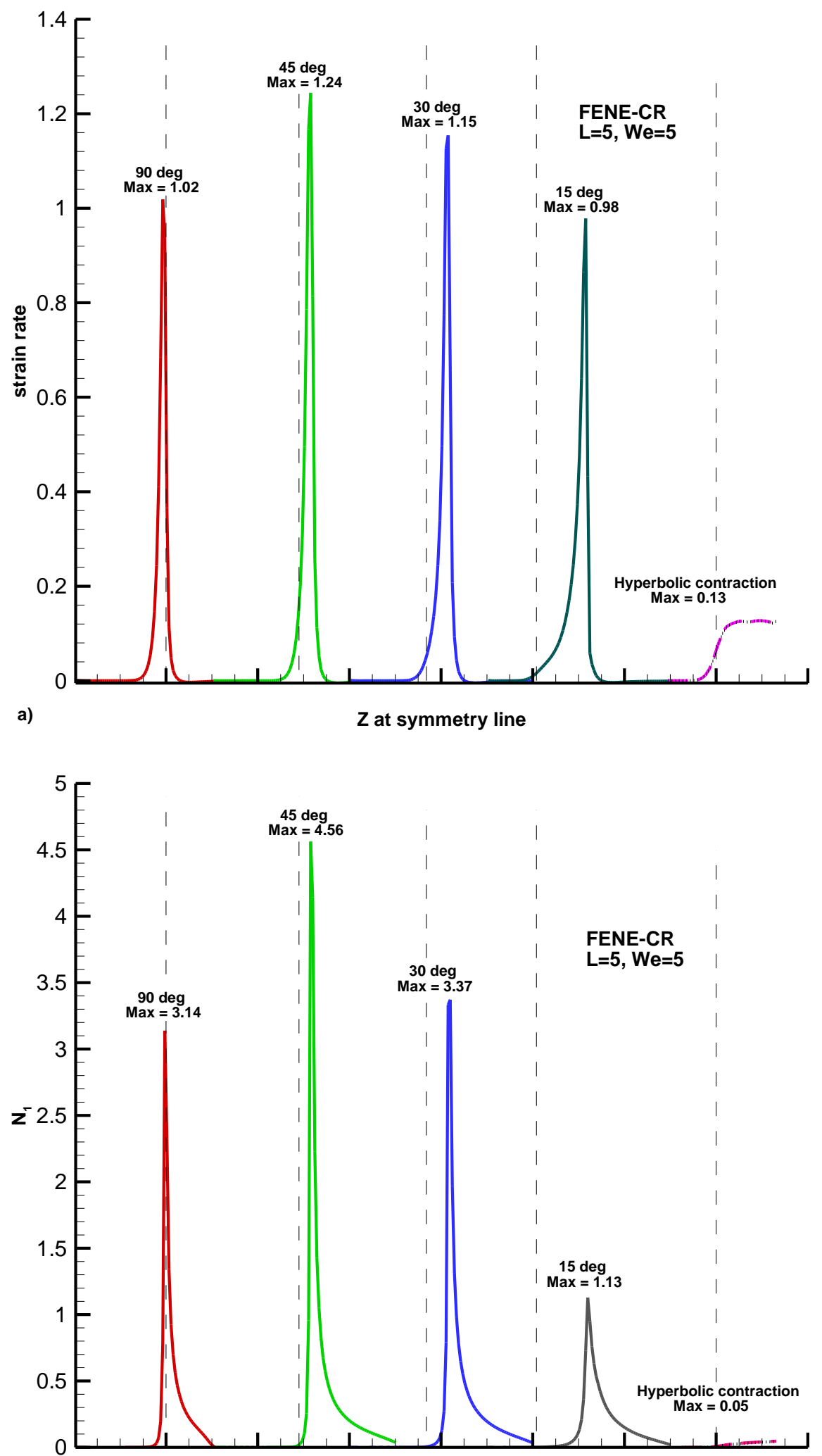

b)

$Z$ at symmetry line 


\section{Figure 11}

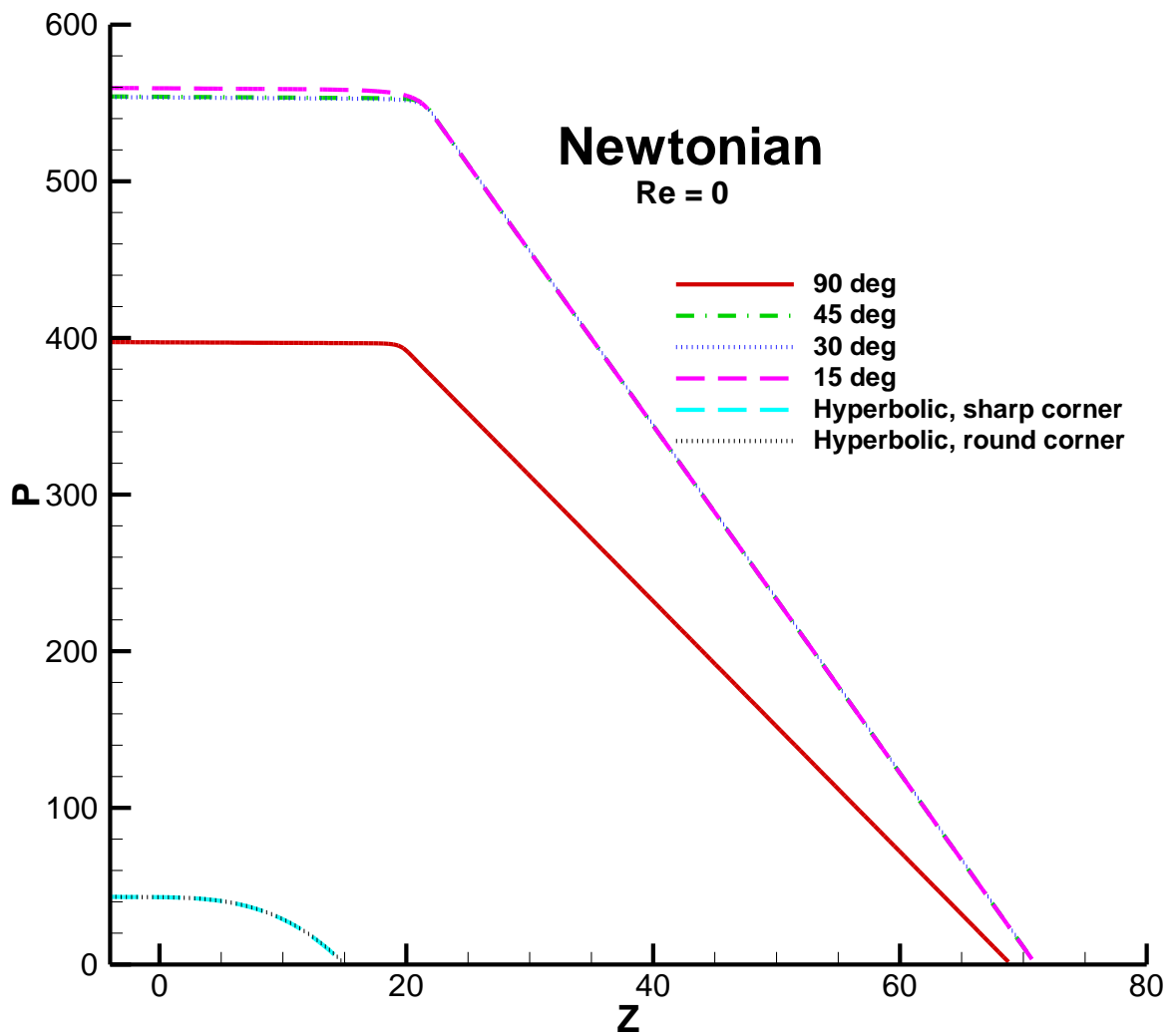


Figure 12

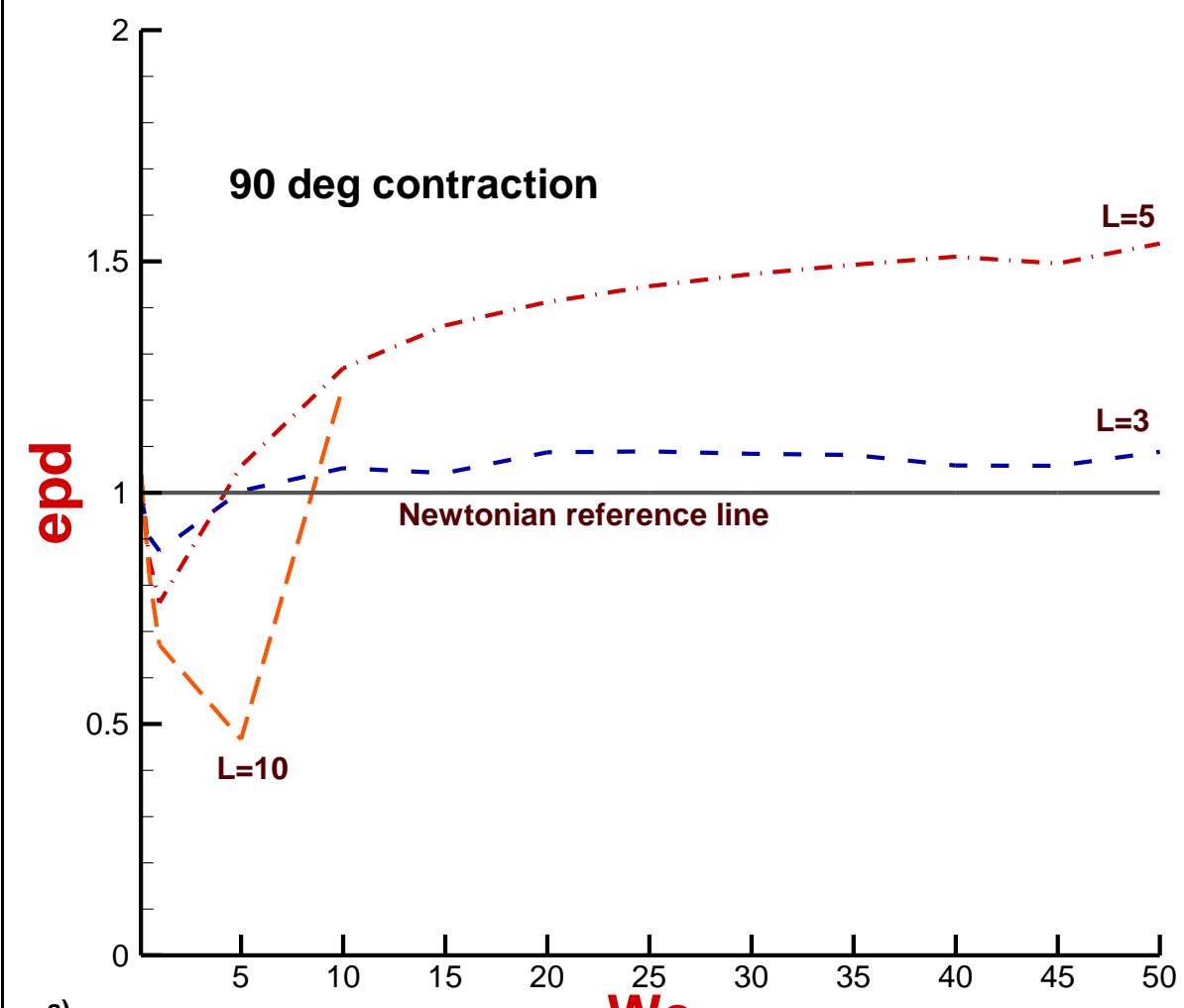

a)

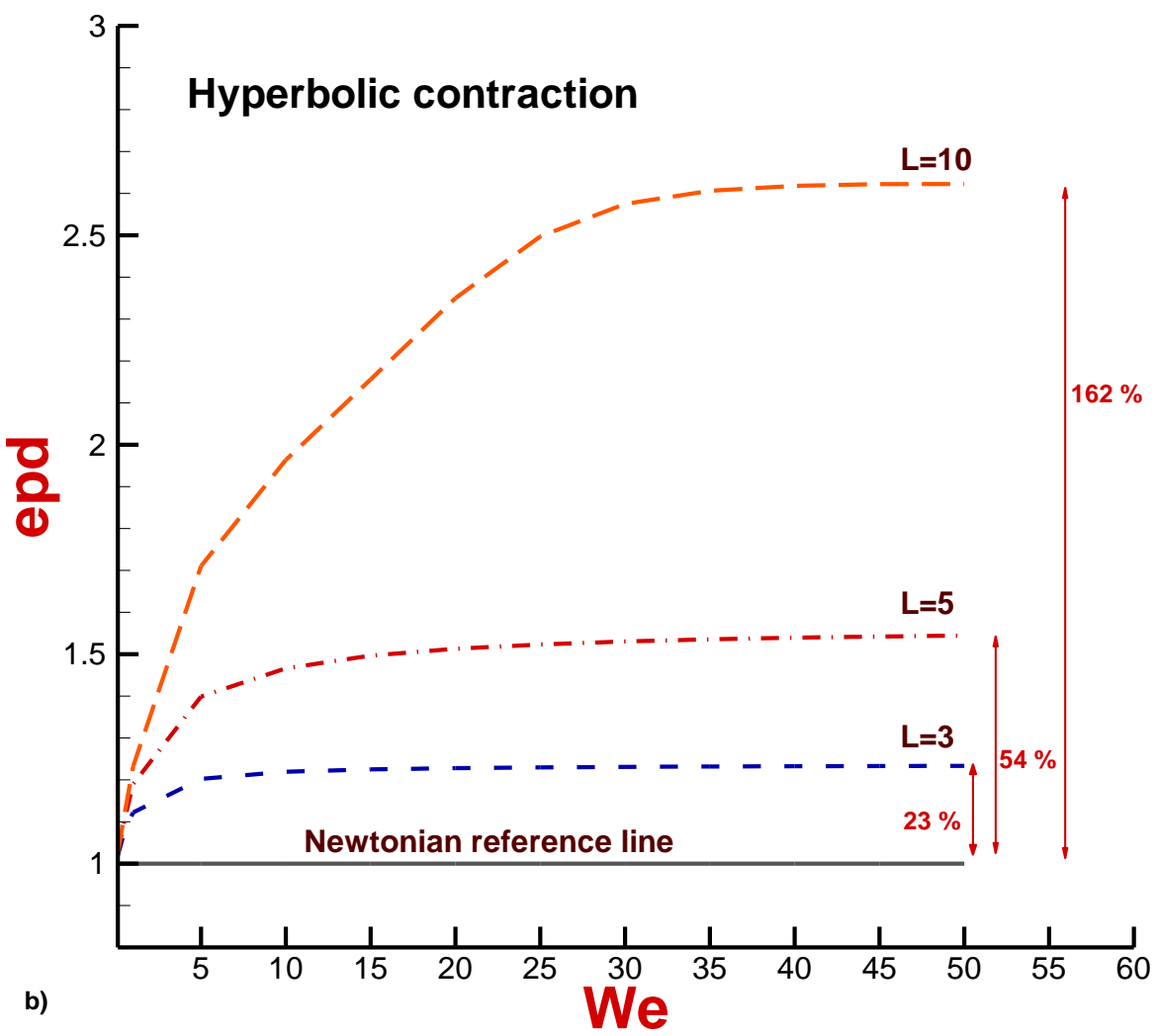

Figure 13 


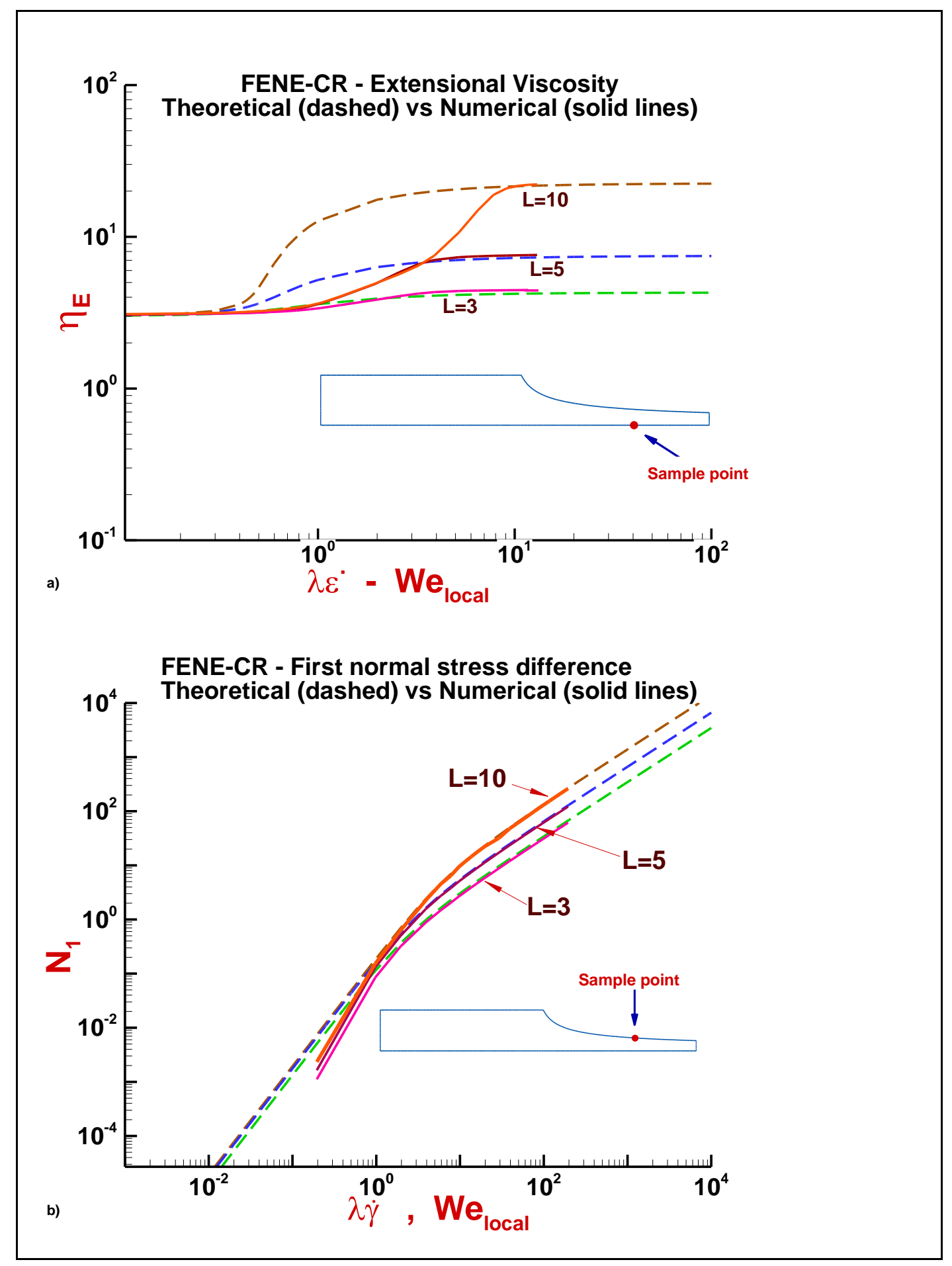

\section{Figure Legend}

Fig. 1 a) First normal stress difference data for FENE-CR model, increasing $L$.

Fig. 1 b) Extensional viscosity data for FENE-CR model, increasing $L$.

Fig. 2. Different geometries used in the study with corresponding meshes. The flow direction for all geometries is from left to right.

Fig. 3. Mesh refinement: Mesh A - coarse; Mesh B - medium; Mesh C - most refined. 
Fig. 4. FENE-CR mesh-refined solutions: a) velocity, b) strain rate along symmetry line for hyperbolic 4:1 contraction with three different mesh densities.

Fig. 5. Stream traces for a Boger fluid with a) 90 degree contraction, $W e=0.1$; b) hyperbolic sharp contraction, $W e=100$; FENE-CR $(L=5)$.

Fig. 6. Strain rate along symmetry line for hyperbolic contraction with sharp (dashed-dotted line) and rounded corner (dashed line) at a) $W e=5$ and b) $W e=1,5$ and 10.

Fig. 7 a) Normal stress difference $\left(N_{l}\right)$ (in extension) along symmetry line for hyperbolic contraction with round (dashed lines) and sharp (dotted lines) corner at $\mathrm{We}=1,5$ and 10 for a Boger fluid.

Fig. 7 b) Normal stress difference $\left(N_{l}\right)$ (in shear) along the geometry wall for hyperbolic contraction with round (dashed lines) and sharp (dotted lines) corner at $\mathrm{We}=1,5$ and 10 for a Boger fluid.

Fig. 8. Shear rate along the wall for hyperbolic contraction with round (dashed lines) and sharp (dotted lines) corner at $W e=5$.

Fig. 9. Contour plots of strain rate (0.1 to 1.2 units) through the different geometries.

Fig. 10. FENE-CR solutions showing the a) strain rate along the symmetry line for different contractions at $L=5, W e=5$. b) Normal stress difference $\left(N_{l}\right)$ along symmetry line for different contractions at $W e=1,5$ and 10 .

Fig. 11. Pressure along symmetry line for the different contractions for a Newtonian case at $R e=0$.

Fig. 12 Excess pressure drop a) 90 degree contraction, b) hyperbolic contraction, $0.1 \leq \mathrm{We}_{\text {local }} \leq$ $10^{2}$, FENE-CR (L=3, 5, 10).

Fig. 13 a) Extensional viscosity, d) First normal stress difference: hyperbolic contraction, theoretical (dashed) vs numerical prediction (solid lines); $0.1 \leq \mathrm{We}_{\text {local }} \leq 10^{2}$, FENE-CR $(\mathrm{L}=3,5$, $10)$.

\section{Tables}

\section{Table 1}

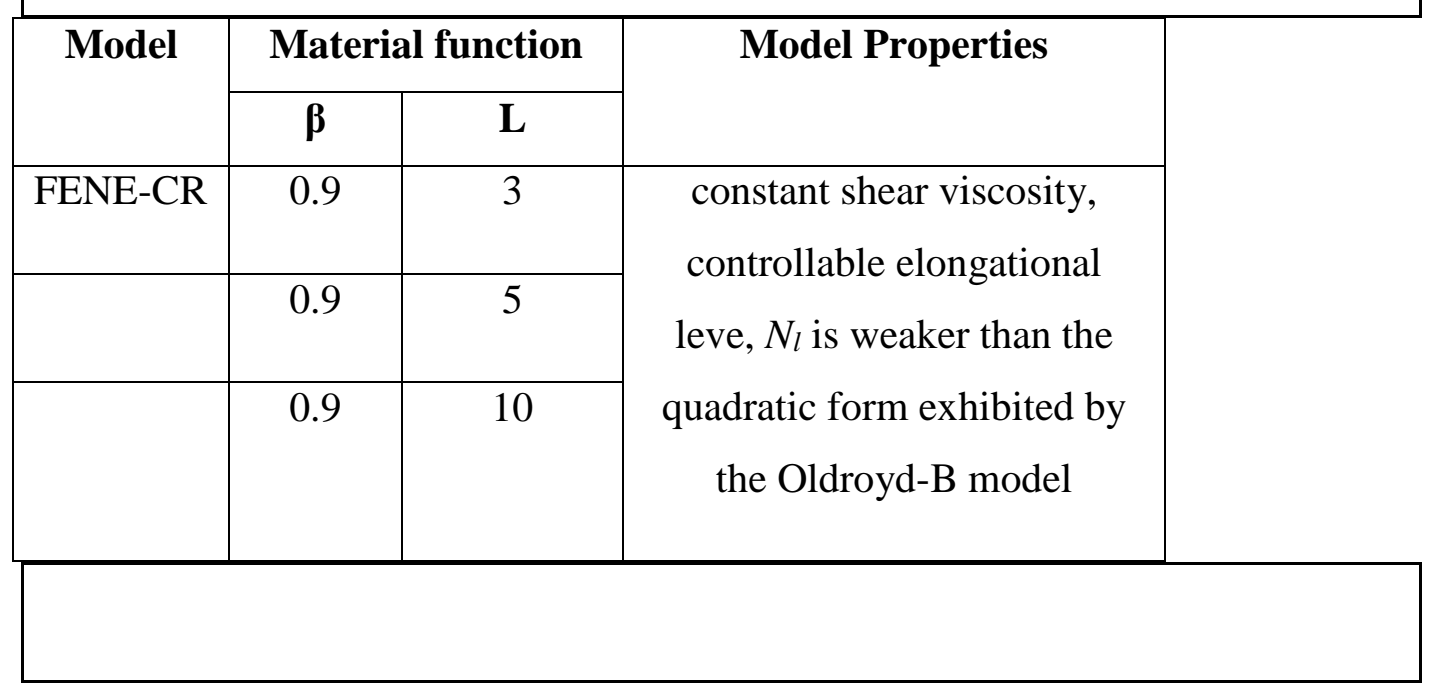




\section{Table 2}

\begin{tabular}{|l|c|c|c|}
\hline Configuration & Elements & Nodes & Degrees of freedom \\
\hline Mesh 90 & 1140 & 2427 & 15206 \\
\hline Mesh 40 & 1245 & 2676 & 16772 \\
\hline Mesh 30 & 1245 & 2676 & 16772 \\
\hline Mesh 15 & 1325 & 2844 & 17824 \\
\hline Hyperbolic sharp & 1350 & 2869 & 17974 \\
\hline $\begin{array}{l}\text { Hyperbolic round } \\
\text { (Mesh A) }\end{array}$ & 1350 & 2869 & 22492 \\
\hline $\begin{array}{l}\text { Hyperbolic round } \\
\text { (Mesh B) }\end{array}$ & 1700 & 3591 & 28333 \\
\hline $\begin{array}{l}\text { Hyperbolic round } \\
\text { (Mesh C) }\end{array}$ & 2160 & 4525 & \\
\hline
\end{tabular}

\section{Table Legend}

Table 1. Boger fluid, material functions for the FENE-CR model.

Table 2. Mesh data 